\title{
Impact of magnetic islands in the plasma edge on particle fueling and exhaust in the HSX and W7-X stellarators
}

L. Stephey, ${ }^{1, a)}$ A. Bader, ${ }^{1}$ F. Effenberg, ${ }^{1}$ O. Schmitz, ${ }^{1}$ G. A. Wurden, ${ }^{2}$ D. T. Anderson, ${ }^{1}$ F. S. B. Anderson, ${ }^{1}$ C. Biedermann, ${ }^{3}$ A. Dinklage, ${ }^{3}$ Y. Feng, ${ }^{3}$ H. Frerichs, ${ }^{1}$ G. Fuchert, ${ }^{3}$ J. Geiger, ${ }^{3}$ J. Harris, ${ }^{4}$ R. König, ${ }^{3}$ P. Kornejew, ${ }^{3}$ M. Krychowiak, ${ }^{3}$ J. D. Lore, ${ }^{4}$ E. A. Unterberg, ${ }^{4}$ I. Waters, ${ }^{1}$ and the W7-X team ${ }^{\mathrm{b}}$ )

1) University of Wisconsin - Madison, Wisconsin 53706, USA

${ }^{2)}$ Los Alamos National Laboratory, New Mexico 87545, USA

${ }^{3)}$ Max-Planck-Institut für Plasmaphysik, 17491 Greifswald, Germany

4) Oak Ridge National Laboratory, Tennessee 37831, USA

(Dated: 27 April 2018)

The edge magnetic structure in the Helically Symmetric eXperiment (HSX) and Wendelstein 7X (W7-X) stellarators has been shown to have a significant impact on the particle fueling and exhaust of the plasma main species (hydrogen) as well as impurity helium. For HSX, the plasma sourcing to exhaust ratio, quantified by the effective and global particle confinement times $\tau_{p}^{*}$ and $\tau_{p, H}$, respectively, increases when a magnetic island chain is located in the plasma edge. The fueling efficiency is reduced by $25 \%$ when the plasma boundary is deformed by the magnetic islands. The X-point geometry also yields higher plasma temperatures in front of the main recycling region. When the island is moved radially inward, both $\tau_{p}^{*}$ and $\tau_{p}$ decrease by $10-25 \%$ depending on plasma density. The $\tau_{p, H}$ results rely heavily on EMC3-EIRENE modeling which confirms reduced fueling efficiency due to more rapid ionization in the outward shifted island position. These findings suggest that for a helically optimized system like HSX, the plasma fueling from the recycling source, as well as from active gas injection, can be controlled by the magnetic island chain in the plasma edge - which is a basic requirement for a divertor system. This process is also effective for the control of effective helium exhaust times, as $\tau_{p, H e}^{*}$ measured by perturbative gas puff experiments is reduced by up to $40 \%$ when the islands are shifted inwards. For Wendelstein 7-X, a similar reduction of $\tau_{p, H e}^{*}$ was inferred when magnetic islands were moved from the far plasma edge into the confined plasma region. However, the effective confinement features of $\mathrm{H}$ as the main plasma species were not affected due to the non-optimal position of the magnetic islands with respect to the highly localized ionization domain during the limiter startup campaign.

\section{INTRODUCTION}

The edge magnetic structure affects particle fueling and exhaust and has an important impact on the impurity handling in both tokamaks as well as stellarators ${ }^{1,2}$. Stellarators (and tokamaks) have magnetic islands when the quantity $\frac{\iota}{2 \pi}(r)$ crosses rational values. The quantity $\frac{\iota}{2 \pi}(r)$, typically used by the stellarator community and henceforth referred to as the "iota profile", is the inverse of $q(r)$, the tokamak safety factor ${ }^{3}$. At rational values of $\frac{\iota}{2 \pi}(\mathrm{r})$, the plasma field lines form magnetic island structures because of harmonic perturbations of the field lines by resonant components in the confining magnetic field ${ }^{4}$. The radial position of these islands can be adjusted by raising or lowering the iota profile, through variations in the magnetic shear, or by introduction of resonant magnetic perturbations (RMPs). In the stellarator edge, however, these islands can be embedded into a chaotic magnetic field structure consisting of regions of long, short, and infinite connection length, $L_{c}$. In this work, $2 L_{c}$ will be defined as the wall-to-wall or target-to-target distance traversed by a magnetic field

\footnotetext{
a) Electronic mail: lastephey@lbl.gov

b) See R. C. Wolf et al., "Major results from the first plasma campaign of the Wendelstein 7-X stellarator," Nucl. Fusion 57, 102020 (2017).
}

line, so the connection length is $L_{c}{ }^{5}$.

It is important to consider that stellarator edges behave differently than tokamaks, even those with $3-\mathrm{D}$ fields. This is mostly due to the difference in rotational transform profiles and the relative strengths of the 3$\mathrm{D}$ shaping fields. In general, unstable manifolds are not considered in stellarator edges and no parallel-to-the-lobe structure, which has been observed on tokamaks with RMPs, has yet been observed on stellarators. For an overview of stellarator edge and divertor design, we recommend the review paper by König et al.: ("Divertor program on stellarators" 6 ) and a paper comparing tokamak and stellarator edges by Feng et al.: ("Comparison between stellarator and tokamak divertor transport"7).

Magnetic islands have been shown to impact the plasma temperature, density, local plasma flow and radial electric field at $\mathrm{LHD}^{8-10}$. LHD measured a flattening of the temperature profile, density peaking within the island, and sheared poloidal flows and radial electric fields at the island boundary. Measurements at HSX have also indicated density and plasma potential peaking in the $8 / 7$ edge island $^{11}$. Additionally, the magnetic island position relative to the ionization region (in which the neutral density profile decays) and the recycling region (in which the neutral profile increases) has been shown to affect helium exhaust and confinement in TEXTOR and $\mathrm{LHD}^{12-14}$. These experiments showed that magnetic islands in the plasma edge led to a reduced helium fuel- 
ing efficiency and also a reduction in the effective helium confinement time in TEXTOR and LHD. It is important to note, however, that these islands were located radially inside the boundary of plasma source region, $S_{p}(r)$, where $S_{p}$ is the ionization source rate in units of ionizations per $\mathrm{cm}^{3}$ per second, and $S_{p}(r)$ denotes that this source rate varies as a function of the minor radius. In these experiments, the impact of the islands on the radial transport and parallel transport balance for impurities, specifically the balance between the friction and thermal force, affects the inward fueling and outward transport ratio $^{12,13}$.

In this work, results are presented from experiments at the Helically Symmetric eXperiment (HSX) ${ }^{15}$ and Wendelstein $7-\mathrm{X}(\mathrm{W} 7-\mathrm{X})^{16}$ stellarators to assess the impact of magnetic islands in the plasma edge region on particle fueling and exhaust. The plasma is treated as a single reservoir for these studies with hydrogen $(\mathrm{H})$ as the main plasma species and helium (He) as a trace impurity. On both devices, the position of a magnetic island chain in the region around the last closed flux surface (LCFS) is varied in an effort to affect plasma and impurity fueling and exhaust. However, while at HSX a change of the radial location of the island was performed with a radial step size comparable to the width of the neutral source profile, at W7-X this type of change was not possible (see Section II for more details).

The paper is organized as follows: in Section II, the two magnetic configurations examined at HSX and W7$\mathrm{X}$ are introduced. In Section III, the experimental measurements and techniques that were used are described. In Section IV, the results for HSX are presented and in Section V, and the results for W7-X are presented. In Section VI, modeling results with the EMC3-EIRENE 3D plasma edge fluid and kinetic neutral transport code are shown as an integral part of the particle balance approach used for both devices. Finally in Section VII, the experimental and modeling results are compared and discussed.

\section{MAGNETIC CONFIGURATIONS EXAMINED AT HSX AND W7-X}

In this work, we assess the impact of the position of magnetic islands at the plasma boundary on plasma fueling and exhaust. The experiments at HSX serve as a foundation for the link between island position and the subsequent impact on the particle balance. This is accomplished by altering the island position relative to the plasma source region. It should be noted that the terms "plasma source region" and "ionization domain" will be used interchangeably throughout the rest of this paper.

The ionization domain $\left(S_{p}(r)\right)$ is the region in which the neutral particles sourced from the limiter, the wall, the divertor, or the gas injection system, are ionized. For regions in which the diffusive transport dominates (notably within the LCFS), it is useful to use the ionization length as a metric. We define the ionization length, $\lambda_{i z}$, as:

$$
\lambda_{i z}=\frac{v_{n}}{n_{e}\left\langle\sigma_{I} v_{e}\right\rangle}
$$

where $v_{n}$ is the thermal velocity of the neutral, $n_{e}$ is the electron density, and $\left\langle\sigma_{I} v_{e}\right\rangle$ is the rate coefficient for electron impact ionization, the dominant ionization process in the plasmas considered in this work (Equation $4.55 \mathrm{in}^{5}$ ).

To categorize the magnetic configurations explored at both HSX and W7-X, we use the location of the magnetic island relative to the main plasma source location. Here, we consider the plasma particle source profile $S_{p}(r)$ which typically peaks near the LCFS. Typical edge and core plasma parameters for HSX and W7-X are summarized in Tables I and II, respectively. Due to lower plasma densities, HSX has a more gradual falloff of the neutral source towards the plasma center as compared to W7-X (i.e. a larger value of $\lambda_{i z}$ ). Despite this, the dominant plasma particle source is located near the LCFS. (This qualitative discussion about $S_{p}(r)$ is based on modeling results with the fully 3 -D plasma edge fluid transport and kinetic neutral gas transport code EMC3-EIRENE, which will be discussed in detail in Section VI.)

It should be noted that the shape and location of $S_{p}(r)$ in the stellarator edge is not static with respect to the magnetic configuration. The experiments performed in this work were not simply a matter of inserting islands into and removing islands from this source region. Rather, as will be discussed in Section VII, the presence of the island can alter the $S_{p}(r)$, and therefore it must be treated in a self-consistent manner.

TABLE I: HSX Parameters

\begin{tabular}{||c|c|c||}
\hline Parameter & Core & Edge \\
\hline \hline $\mathrm{n}_{e}$ & $4 \mathrm{E} 12 \mathrm{~cm}^{-3}$ & $1 \mathrm{E} 11 \mathrm{~cm}^{-3}$ \\
$\mathrm{~T}_{e}$ & $2.5 \mathrm{keV}$ & $70 \mathrm{eV}$ \\
$\mathrm{T}_{i}$ & $60 \mathrm{eV}$ & $25 \mathrm{eV}$ \\
\hline
\end{tabular}

TABLE II: W7-X Parameters

\begin{tabular}{||c|c|c||}
\hline Parameter & Core & Edge \\
\hline \hline $\mathrm{n}_{e}$ & $4 \mathrm{E} 13 \mathrm{~cm}^{-3}$ & $1 \mathrm{E} 12 \mathrm{~cm}^{-3}$ \\
$\mathrm{~T}_{e}$ & $10 \mathrm{keV}$ & $40 \mathrm{eV}$ \\
$\mathrm{T}_{i}$ & $1 \mathrm{keV}$ & no data \\
\hline
\end{tabular}

Two different magnetic configurations were examined in each device: one in which the magnetic island was located in the increasing section of $S_{p}(r)$ (outside the LCFS), and one in which the island was moved inward to be located in the decreasing section of $S_{p}(r)$ (inside the LCFS). At HSX, this manipulation of the island position inwards did change the $S_{p}(r)$ profile itself, but the resulting changes were small enough that the $8 / 7$ island 
chain could still be shifted from one side of the source profile to the other.

At W7-X, however, the change in the iota profile was only able to move the $m / n=5 / 5$ island chain from outside the vessel into the scrape-off layer, while at the same time moving the $m / n=5 / 6$ island chain from $1-2 \mathrm{~cm}$ inside the LCFS to $10 \mathrm{~cm}$ inside the LCFS. This restriction was due to commissioning and engineering constraints. Thus in neither configuration could the island be placed in the peak $S_{p}(r)$ region, and therefore a less pronounced effect might be expected. It should also be noted that in each of these configuration pairs, an effort was made to preserve the general and core transport properties by keeping the plasma volume, density, temperature (and neoclassical transport, through the effective ripple, $\epsilon_{\mathrm{eff}}{ }^{17}$, as close as possible). As a result we expect that any changes we observe in the particle penetration and confinement properties should stem primarily from the edge magnetic topology changes.

A summary of the four magnetic configurations explored in this work is shown in Fig. 1. This figure visualizes the relative relationship between the magnetic island chain(s) and the EMC3-EIRENE flux surface averaged ionization domain in each configuration. The EdgeIslands and No-Edge-Islands configurations at HSX are pictured in (a) and (b), respectively. The Increased Iota configuration and the Standard configuration are pictured in (c) and (d), respectively. It should be noted that all simulations and calculations have been performed using the vacuum magnetic field (i.e. the value of $\beta$, the ratio between the plasma pressure and the magnetic pressure $^{3}$, is known to be very small for all configurations at HSX and W7-X examined in this work $\left.{ }^{18,19}\right)$. Thus the vacuum approximation is sufficient for the analyses in this study.

The two magnetic configurations assessed at HSX are shown at the top of Fig. 2. The configuration in which the magnetic islands are located in the plasma edge, i.e. outside of the main ionization region (called the EdgeIslands configuration) is shown in Fig. 2(a). It is also known as the QHS (quasi-helical symmetry) configuration in other HSX publications. In this configuration, the intrinsic 8/7 magnetic island chain resides immediately outside the LCFS. The configuration in which the islands are shifted inwards and reside inside of the maximum of $S_{p}(r)$ (called the No-Edge-Islands configuration) is shown in Fig. 2(b). This magnetic configuration of HSX is part of the magnetic well-type configuration family, and in this configuration the iota profile has been raised to move the $8 / 7$ island chain inside the LCFS. In both plots, the Poincare plot for each configuration is shown with black dots, and the colored regions show the edge connection length structure with the longest connection lengths shown in red. Note that at HSX no limiters were present. As a result, because the edge field lines and the $8 / 7$ islands partially intersect the wall, a complex stochastic structure is present in the Edge-Islands configuration. The effective ripple in the No-Edge-Islands con- figuration is approximately a factor of two larger than the Edge-Islands configuration. Both the iota profile and the effective ripple for both configurations are shown in Fig. 3(a) and (b), respectively.

Fig. 4 shows Thomson scattering data from both magnetic configurations in HSX. Data from the Edge-Islands configuration are shown in red and data from the NoEdge-Islands configuration are shown in gray. These Thomson data are determined from the mean of two time-points and the error bars represent the combined error of both individual time-points. The most marked difference between the two configurations appears inside of $\mathrm{r} / \mathrm{a}=0 . \sim 2$.

The magnetic structure of the two configurations assessed at W7-X is shown at the bottom of Fig. 2. The socalled Increased Iota configuration is shown in Fig. 2(c) and the Standard configuration is shown in Fig. 2(d). In both plots, the Poincaré plot for each configuration is shown with red dots, and the colored regions show the edge connection length structure with the longest connection lengths shown in white. To create the Increased Iota configuration, the iota profile was raised to move the $5 / 6$ chain further inside the LCFS, and to introduce the $5 / 5$ island chain into the far SOL. The Increased Iota configuration has an effective ripple, $\epsilon_{\text {eff }}$, approximately a factor of two greater than that in the Standard configuration. The iota profiles and $\epsilon_{\text {eff }}$ profiles for both configurations are shown in Fig. 3.

Thomson profile data from the two W7-X magnetic configurations are shown in Fig. 5. Data in the Increased Iota configuration are shown in green and data in the Standard configuration are shown in blue. These profiles are fits generated from the measurement points. The shaded regions above and below the fit lines indicate the 95 percent confidence region. Unlike at HSX, these measurements suggest that the density and temperature profiles appear to be very similar between the two configurations despite the increase in $\epsilon_{\text {eff }}$ of approximately a factor of two.

\section{MEASUREMENT AND ANALYSIS APPROACH ON BOTH DEVICES}

In this section we provide a brief overview of the analysis methods of measurements used to assess changes in particle fueling and confinement at HSX and W7-X as a result of changing the edge magnetic structure. We will first describe the two main types of measurements used: the effective particle confinement time $\tau_{p}^{*}$ and the actual global particle confinement time $\tau_{p}$, based on a single reservoir particle balance. We will then provide a more detailed overview of the principal diagnostics and measurements used in this work at both HSX and W7-X. 

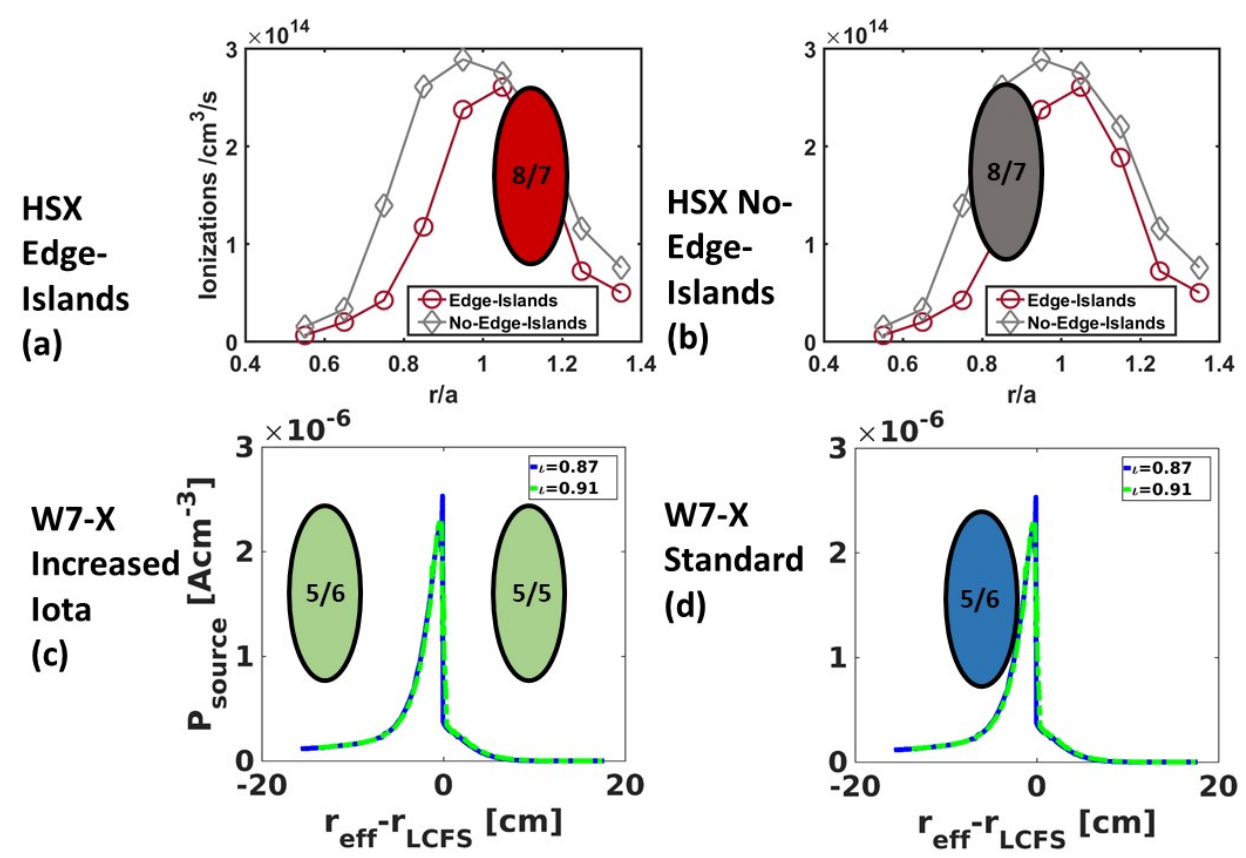

FIG. 1: A summary of the relationship between the island position and the ionization source region for the four configurations that have been explored in this work. The flux surface averaged ionization rate plots are repeated from earlier figures. The approximate location of the island chains have been noted with oval cartoons superimposed on the ionization domain plots. The magnetic island position(s) relative to the ionization domain have been noted for both the HSX configurations ( $a$ and b) and the W7-X configuration (c and d).

\section{A. Effective particle confinement time}

The effective particle confinement time $\tau_{p}^{*}$ is the decay time of some particle-confinement related quantity, usually plasma density or spectroscopic emission. For the situation where the majority of the plasma source comes from recycling (i.e. wall or limiter recycling), the effective particle confinement time is connected to the global confinement time $\tau_{p}$ of a reservoir by the recycling coefficient $R$ :

$$
\tau_{p}^{*}=\frac{\tau_{p}}{1-R}
$$

$\tau_{p}^{*}$ is usually determined by fitting the decay of the plasma density following a perturbative gas injection. This gas injection provides a transient particle source to the plasma and after it ceases, the decay of the density spike from this gas injection can be measured. For the case of perturbative gas injection experiments with $\mathrm{He}$, it is assumed that the emission of the He-II line at $\lambda=468.6$ $\mathrm{nm}$ is representative, to first order, of the singly ionized helium density and He-I emission at $\lambda_{1}=667.8 \mathrm{~nm}$ or $\lambda_{2}=587.4 \mathrm{~nm}$ are representative, again to first order, for the helium neutral density. Measuring $\tau_{p}^{*}$ by such perturbative gas injections is a commonly used technique ${ }^{12,20,21}$ to infer effective confinement properties of the measured species.

\section{B. Global particle confinement time}

In order to establish a balance between the total number of particles $N_{\text {tot }}$ and its time rate of change, $d N_{\text {tot }} / d t$, in the plasma reservoir due to particle fueling rates and particle exhaust quantities, a single reservoir particle balance method is used. To construct this balance, the plasma is treated as the reservoir, and all particles inside, entering, and exiting the reservoir must be quantified. Such 0-D single reservoir models are commonly used $^{22-24}$. Below is the formulation of this 0-D balance employed in this study:

$$
\begin{array}{r}
\frac{d N_{\text {tot }}}{d t}= \\
-\frac{N_{\text {tot }}}{\tau_{p}}+f_{\text {gas }} \Phi_{\text {gas }}+f_{\text {wall }} \Phi_{\text {wall }}+f_{\text {lim }} \Phi_{\text {lim }}= \\
\Phi_{\text {ext }}-\Phi_{\text {exhaust }}
\end{array}
$$

where $N_{\text {tot }}$ is the total number of plasma particles, $\tau_{p}$ is the particle confinement time, $f_{\text {gas }}, f_{\text {wall }}$, and $f_{\text {lim }}$ are fueling efficiency coefficients, and $\Phi_{\text {gas }}, \Phi_{\text {wall }}, \Phi_{\text {lim }}$ are particle fluxes from the gas valve, recycling from the main wall, recycling from the limiter and $\Phi_{\text {ext }}$ is the total external fueling, and $\Phi_{\text {exhaust }}$ is the exhaust rate. This will yield $\tau_{p}$ as a characteristic global dwell time as the solution to this 1st order differential equation in the time domain. This characteristic time scale is treated as the global particle confinement time $\tau_{p}$ inside of the reservoir. 


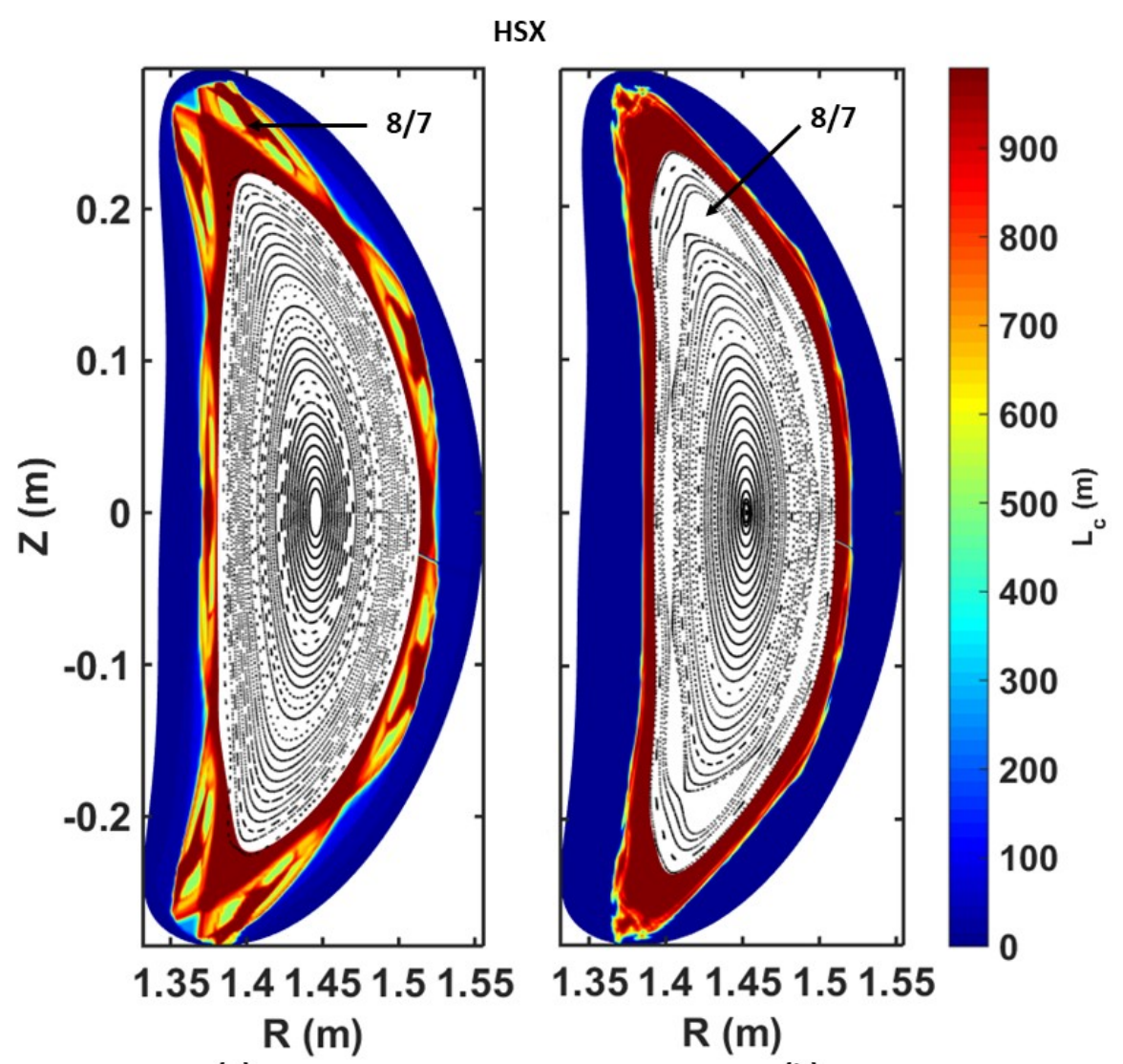

(a)

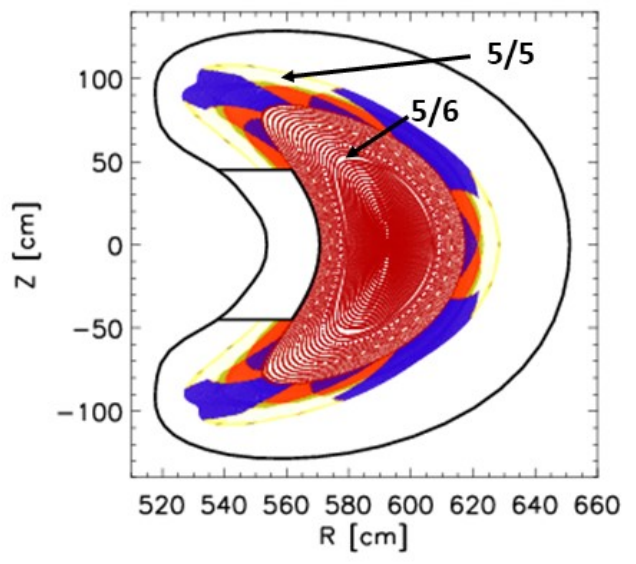

(c)
W7-X
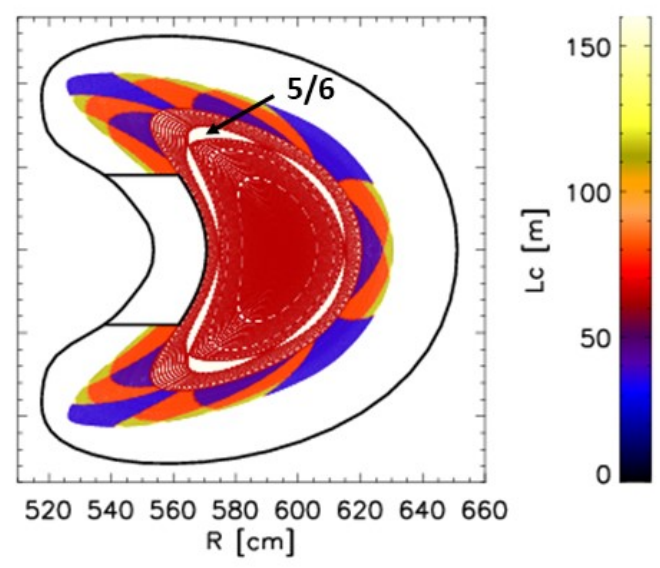

(d)

FIG. 2: (Top figures) Overlay of connection length calculations (colored regions) with Poincaré plots (black dots) for the HSX configurations investigated in this study: the Edge-Islands (a) configuration and the No-Edge-Islands configuration (b). The 8/7 island chain is located outside of the LCFS in the Edge-Islands configuration and is located inside the LCFS in the No-Edge-Islands configuration. (Bottom figures) Overlay of connection length calculations (colored regions) with Poincaré plots (red dots) for the W7-X configurations investigated in this study: the Increased Iota configuration (c) and the Standard configuration (d). In the Standard configuration (d), the 5/5 islands are small and radially far beyond the limiters; the 5/6 island chain can be seen several cm inside the limiter position. In the Increased Iota configuration (c), the $5 / 6$ island has been moved radially inward and subsequently compressed; additionally the $5 / 5$ island has be moved radially inward and now appears in the far SOL. It is shown in white (i.e. infinite) connection lengths. 

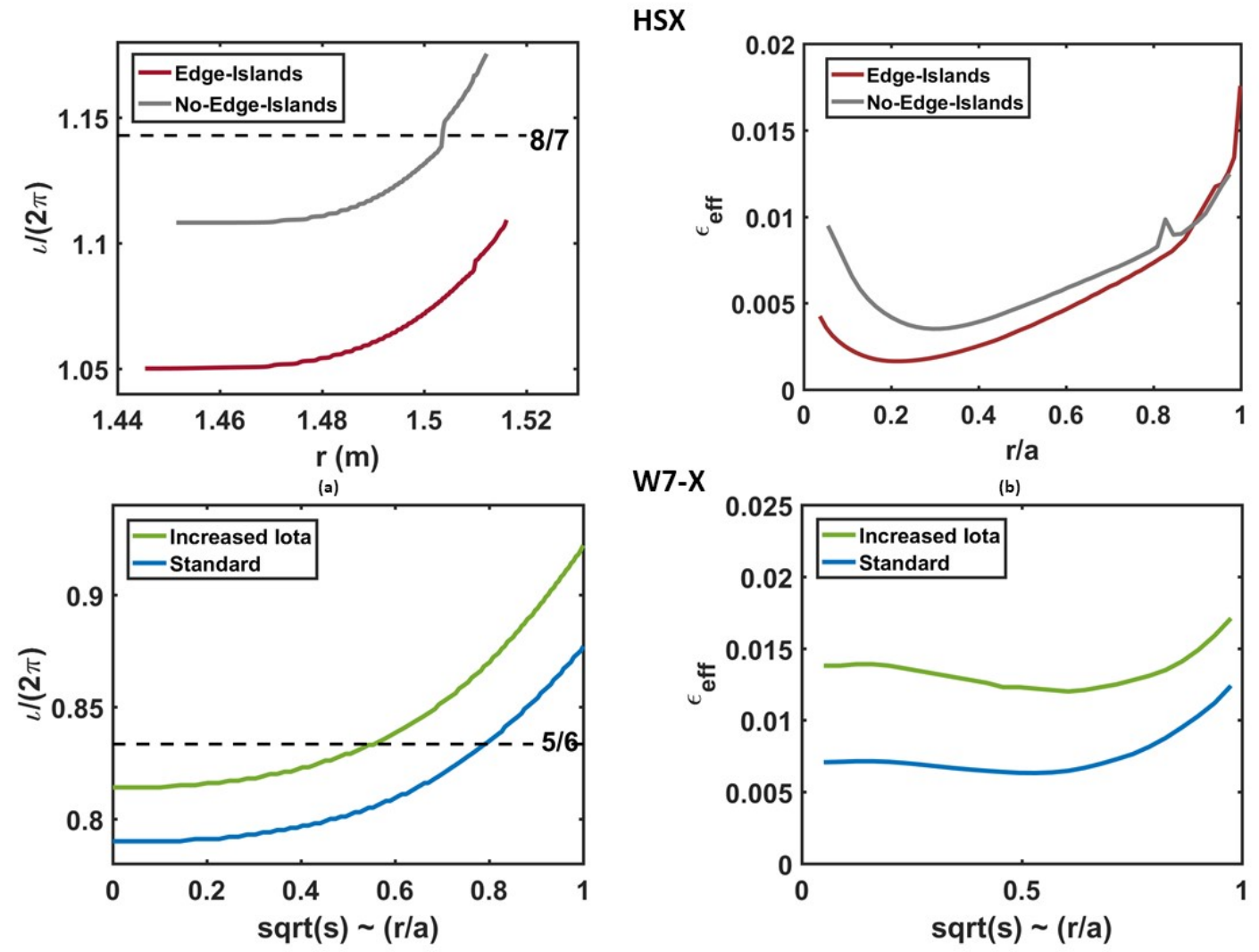

(c)

FIG. 3: (Top left) The rotational transform profile in the Edge-Islands configuration (red) and the No-Edge-Islands configuration (gray). Note the location of the $8 / 7$ resonance. (Top right) The effective ripple in the Edge-Islands configuration (red) and the No-Edge-Islands configuration (gray). (Bottom left) The rotational transform profile in

the Standard configuration (blue) and the Increased Iota configuration (green). Note the location of the 5/6 resonance. (Bottom right) The effective ripple in the Standard configuration (blue) the Increased Iota configuration (green).
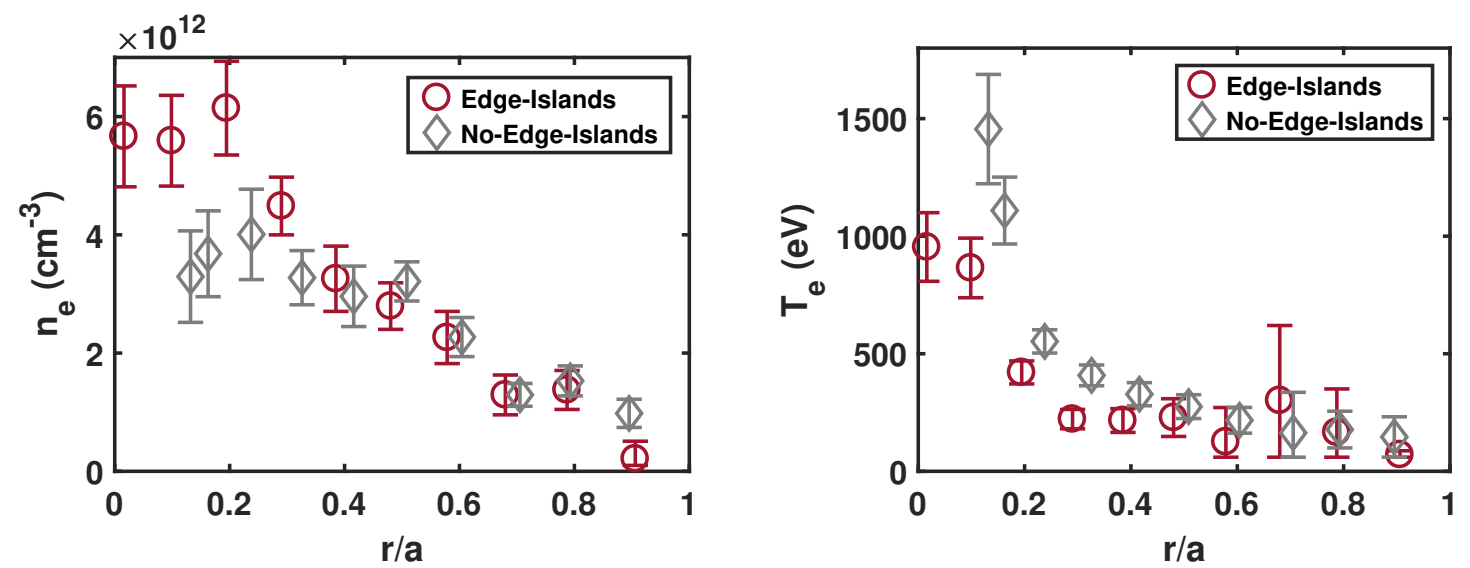

FIG. 4: HSX Thomson scattering profiles from both the Edge-Islands (red circles) and the No-Edge-Islands (gray diamonds) configurations. (Left) Thomson $\mathrm{n}_{e}$ profiles and (Right) Thomson $\mathrm{T}_{e}$ profiles. These profiles were generated from data at two time points $(\mathrm{t}=0.83$ and $\mathrm{t}=0.84 \mathrm{~ms})$ and the error bars represent the sum in quadrature of the error from each individual measurement. Where appropriate, the error bars were constrained to lie within physically realistic values. The shift of the magnetic axis in the No-Edge-Islands configuration results in decreased core resolution. 

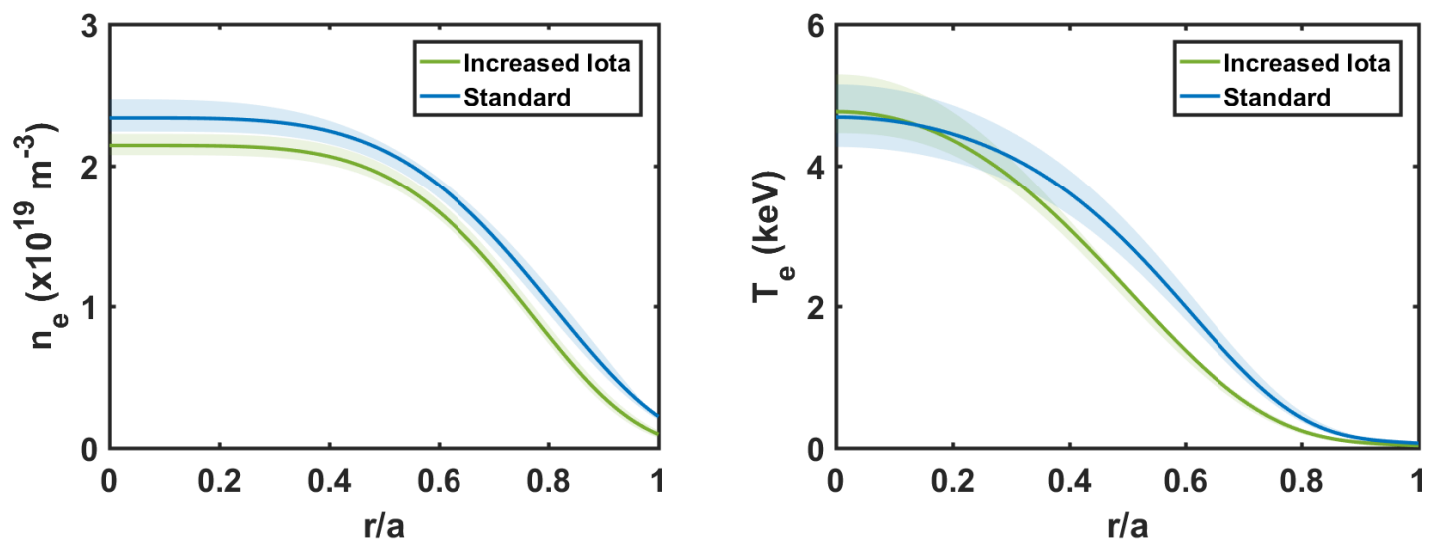

FIG. 5: Thomson scattering measurements in the W7-X Increased Iota configuration (green) and in the Standard configuration (blue). The density profile is shown on the left and the temperature profile is shown on the right. The solid lines are fit lines to the measured data points. The shaded regions represent the 95 percent confidence error bars for each fit.

This balance includes information about fueling efficiencies (the $f$ coefficients) for a given particle source/sink condition and plasma regime. The fueling efficiency is defined as the fraction of total ionizations that occur within the confinement region:

$$
f_{\text {eff }}=\frac{S_{\mathrm{p}, \text { conf }}}{S_{\mathrm{p}, \text { conf }}+S_{\mathrm{p}, \mathrm{SOL}}}
$$

where $f_{\text {eff }}$ is the fueling efficiency coefficient, $S_{\mathrm{p}, \text { conf }}$ is the ionization source rate inside the confinement region, and $S_{\mathrm{p} \text {,conf }}$ is the ionization source rate outside the confinement region (i.e. in the SOL). In particular, the fueling efficiency depends on the ionization scale length $\lambda_{i z}$ (see Equation 1), which is a function of the background plasma temperature and density. These fueling efficiencies are obtained from EMC3-EIRENE modeling and will be discussed in more detail in Section VI.

Additionally, the particle balance relies on the accurate measurement of the recycling flux terms from wall and limiter surfaces. However, due to the 3-D shape of the vessel wall and the fact that the entire surface area of the wall could not be observed with suitable cameras or other diagnostics, extrapolations of local measurements are required to obtain these major source terms in the particle balance. This is an issue for the absolute accuracy of the extracted values of $\tau_{p}$. However, if the general wall and limiter flux structure does not change substantially between configurations, this uncertainty can instead be treated as a systematic uncertainty which will in turn cancel out when performing relative comparisons between two configurations. Thus the uncertainty in relative changes $\Delta \tau_{p}$ between configurations will have a lower value, defined only by the individual uncertainty in the measurements, which will be discussed in more detail in Sections IV and V.

\section{EXPERIMENTAL STUDIES OF PARTICLE BEHAVIOR AT HSX}

In this section, the key measurements and techniques to study the particle fueling and exhaust properties of the Edge-Islands and No-Edge-Islands configurations at HSX are presented. Discussions and conclusions from these data will be presented in section VII along with results from the W7-X study. The main measurements used in this work are line averaged density $\overline{n_{e}}$ measurements from an interferometer ${ }^{25}$, density $n_{e}(r)$ and temperature $T_{e}(r)$ profiles from Thomson scattering ${ }^{26}$ and a filtered photodiode array system ${ }^{27}$ to measure line emission of Balmer- $\alpha\left(\lambda_{\alpha}=656.3 \mathrm{~nm}\right)$ as line integrated signal at nine radial intersection points. The system also has been upgraded with additional helium channels to measure He-I and He-II line emission profiles for $\tau_{p, H e}^{*}$ measurements.

\section{A. Effective particle confinement time measurements in HSX}

Measurements of $\tau_{p, H}^{*}$ and also of $\tau_{p, H e}^{*}$, discussed below, were obtained by perturbative gas injection experiments. A short injection of the test gas species $(\mathrm{H}$ and $\mathrm{He}$ ) was launched into an equilibrated hydrogen plasma. The exponential decay of electron density after the termination of the gas injection was measured and used as effective confinement time $\tau_{p}^{*}$.

At HSX, a fast valve was used to make a single $6 \mathrm{~ms}$ helium or hydrogen injection of $\sim 1 \times 10^{18}$ particles into a hydrogen plasma. A filtered photodiode array was used to measure decay in He-II emission (468.6 nm), and an interferometer ${ }^{28}$ was used to measure the decay in the line-averaged density for hydrogen injection. The $\mathrm{H}_{\alpha}$ line emission was not used because the decay time was too fast for the photodiodes to resolve differences outside the 


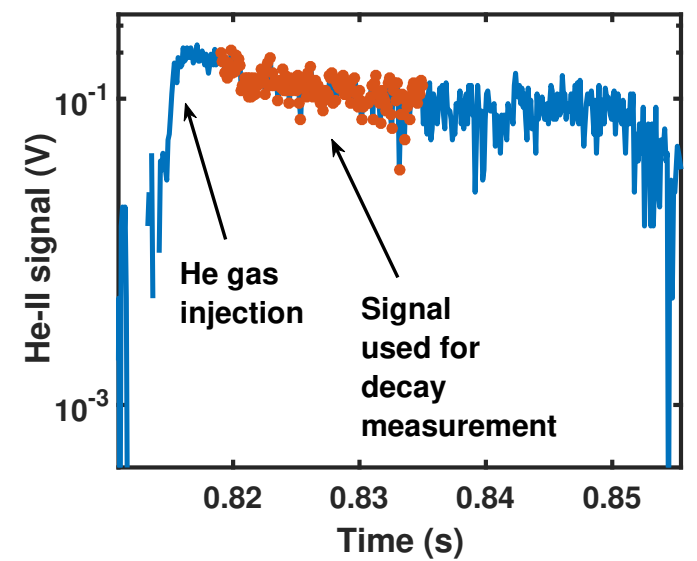

FIG. 6: A representative He-II emission trace from HSX showing the helium gas injection into a hydrogen plasma at $0.81 \mathrm{~s}$ and the portion of the signal that was used to determine the exponential decay (highlighted in orange). Note that this trace was produced by subtracting a reference trace from a shot with no helium injection to remove the contribution of the residual HSX helium source.

measurement uncertainty. For both $\mathrm{H}$ and He injections, the signal from an immediately preceding or following reference discharge was subtracted from the signal obtained during the gas injection discharge. This allowed the contribution from the plasma or He-II background to be removed when determining the decay time. This subtraction was not possible with W7-X data, as will be discussed in Section $\mathrm{V}$ because no intermediate reference discharges were obtained.

Measurements of $\tau_{p, \mathrm{H}}^{*}$ and $\tau_{p, \mathrm{He}-\mathrm{II}}^{*}$ are shown in Fig. 7 . The decay time is longer in the Edge-Islands configuration for both hydrogen and helium injections. In general, $\tau_{p, \mathrm{He}-\mathrm{II}}^{*}$ is a factor of approximately 2 longer than $\tau_{p, \mathrm{H}}^{*}$, most likely due the fact that some fraction of hydrogen is absorbed by the wall for the short duration of HSX discharges while helium is fully recycling and re-enters the plasma at a higher $\operatorname{rate}^{29}$. It should be noted that the error bars shown in Fig. 7 are the uncertainties in the 95 percent confidence exponential fit for each individual data point. The shaded region indicates the mean standard deviation of all the data points from the fit line. A single term power series fitting of the form $y=a x^{b}$ was used the generate a fit line, also plotted.

\section{B. Particle confinement time in HSX}

As discussed in Section III(B), experimental data will be used to infer the particle balance in order to determine the global particle confinement time $\tau_{p}$. Equation 3 shows a generic version of this balance reordered to solve for $\tau_{p}$. For the case of HSX, several terms can be neglected. Because no limiters are inserted for the ex-
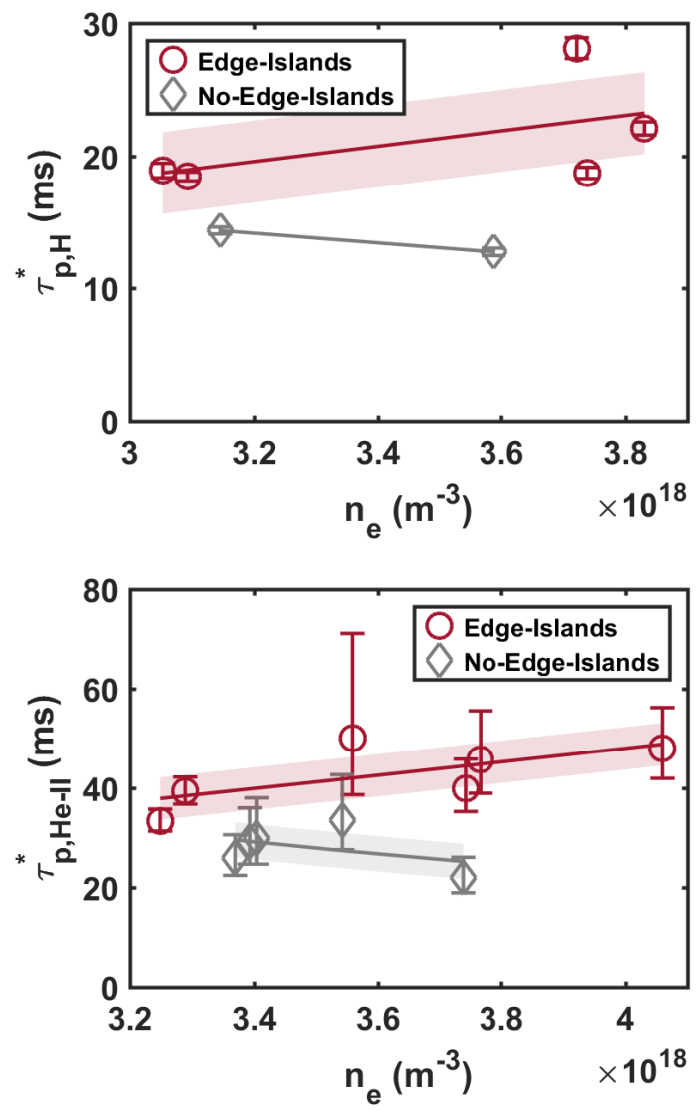

FIG. 7: Figure showing $\tau_{p}^{*}$ measured in HSX in both the Edge-Island (red circles) and No-Edge-Island (gray diamonds) configurations. (Left) $\tau_{p, \mathrm{H}}^{*}$ as a function of $\mathrm{n}_{e}$ (Right) $\tau_{p, \mathrm{He}-\mathrm{II}}^{*}$ as a function of $\mathrm{n}_{e}$. Data are plotted as function of mean $\mathrm{n}_{e}$ determined from the central chord of the HSX interferometer. A single term power series fitting was used the generate a fit line, also plotted. The shaded region indicates the mean standard deviation of the measured data points from the fit line.

periments under consideration, the recycling term at the limiters is not included (i.e. $\Phi_{\text {lim }}=0$ ). The recycling coefficient can be obtained using the effective particle confinement time and the true particle confinement time, which will be discussed at the end of this section. Based on these assumptions, the following formulation for the HSX particle balance is used for the analysis presented:

$$
\tau_{p}=\frac{N_{\mathrm{tot}}}{-\frac{d N_{\text {tot }}}{d t}+f_{\text {gas }} \Phi_{\text {gas }}+f_{\text {wall }} \Phi_{\text {wall }}}
$$

Measurements of the line-averaged density were necessary to determine the following quantities that will later be used: total number of plasma particles, $\mathrm{N}_{\text {tot }}$, which is a product of the line-averaged density $\bar{n}_{e}$ and the confinement volume, $V_{\text {confinement }}$, and the time-derivative of the change in total number of particles, $\frac{d N_{\text {tot }}}{d t}$. Under steady-state conditions, $\frac{d N_{\text {tot }}}{d t}=0$. If conditions are not 
steady-state, $\frac{d N_{\text {tot }}}{d t}$ can be included as necessary. This is the general form of each of the terms that will be used in the particle balance analysis (Equation 3):

$$
N_{\text {tot }}=\overline{n_{e}} V_{\text {confinement }}
$$

and

$$
\frac{d N_{\text {tot }}}{d t}=\frac{d \bar{n}_{e}}{d t} V_{\text {confinement }}
$$

It is important to note that the line-averaged density $\overline{n_{e}}$ is averaged over the 1-D interferometer path length, and is not a true volume-averaged density. Therefore it may overestimate the total number of particles since $\overline{n_{e}}$ will over-emphasize the contribution of the high-density but small volume core. In general this overestimation will be systematic between magnetic configurations. This same procedure is used at both HSX and W7-X since this is the only density measurement available for every plasma discharge analyzed in this work.

The configuration volumes $V_{C}$ require a plasma equilibrium reconstruction. The canonical HSX reference values in $^{30}$ were used. They are $0.378 \mathrm{~m}^{3}$ in the EdgeIslands configuration and $0.356 \mathrm{~m}^{3}$ in the No-EdgeIslands configuration. The uncertainties for the calculations of $V_{C}$ are small ( $\sim 1$ percent) and hence it is assumed that the most significant uncertainty is the standard deviation of the interferometer signal during the analysis window $(\sim 2-5$ percent $)$.

At HSX, the injected gas flux $\Phi_{\text {gas }}$ was determined through a 2-D calibration matrix. Given the voltage signal sent to the piezo electric gas valve and the backing pressure of the gas plenum, the total throughput in a 60 ms pulse could be calculated. (Fueling in HSX typically starts approximately $10 \mathrm{~ms}$ before ECRH initiation and continues through the $50 \mathrm{~ms}$ discharge.) $\Phi_{\text {gas }}$ is calculated directly using the calibration table, and $\Delta \Phi_{\text {gas }}$ is 4 percent based on the measurement uncertainty in the calibration.

The HSX wall particle flux, $\Phi_{\text {wall }}$ :

$$
\Phi_{\text {wall }}=c_{H_{\alpha}} \alpha_{D E G A S} I_{H_{\alpha}}
$$

where $c_{H_{\alpha}}$ is an absolute calibration factor, $\alpha_{D E G A S}$ is the scale factor that matches simulated $H_{\alpha}$ emission to measured $H_{\alpha e m i s s i o n}$ (this will be described in detail in what follows), and $I_{H_{\alpha}}$ is the measured $H_{\alpha}$ intensity signal, was by far the most complex term to obtain for the HSX particle balance. This quantity was determined through a combination of experimental spectroscopic measurements and neutral particle modeling using the DEGAS code ${ }^{31}$. The process was as follows: an array of absolutely calibrated $\mathrm{H}_{\alpha}$ detectors ${ }^{27}$ was used to view the HSX plasma at a location toroidally far away from the gas puff to measure the intensity signal $I_{H_{\alpha}}$. This signal was obtained as a voltage signal $V_{H_{\alpha}}$ from the photodiode and converted into an intensity signal $I_{H_{\alpha}}$ with units [photons $\mathrm{s}^{-1} \mathrm{~cm}^{-2}$ ] using an absolute calibration factor $c_{H_{\alpha}}$.
The DEGAS code, a 3-D Monte-Carlo neutral particle code ${ }^{31}$, was used to simulate the wall-sourced neutral particles in HSX. Only the wall-sourced particles were simulated in an effort to isolate the contribution of the $\Phi_{\text {wall }}$ term to the particle balance. (For reference the gas puff flux $\Phi_{\text {gas }}$ is of the same order as $\Phi_{\text {wall }}$.) The locations of the plasma sources were determined through field line following; the location at which the field line intersected the vessel wall was treated like a source. In addition, a linear relationship between the source rate and the field line connection length was assumed based on previous work $^{32}$. The total source rate from the wall surface area is $\Phi_{\text {wall }}$.

Given a static plasma background determined from the Thomson profiles shown in Fig. 4, the DEGAS code calculates the neutral particle density and the estimated $\mathrm{H}_{\alpha}$ emission rate $I_{H_{\alpha}}$ in each volumetric grid cell. This calculated emission can then be directly compared to the measured $\mathrm{H}_{\alpha}$ emission in a manner similar to that described $\mathrm{in}^{33}$. The scale factor required to match the DEGAS emission to the measured emission is then used to scale the wall particle source rate, which provides the final value of $\Phi_{\text {wall }}$ required for Equation 5 . This scale factor is $\alpha_{D E G A S}$ in Equation 8. The advantage to using DEGAS for this portion of the study is that it includes a package which automatically calculates the expected $\mathrm{H}_{\alpha}$ emission given the neutral density and background plasma parameters, therefore facilitating comparisons with the absolutely calibrated $\mathrm{H}_{\alpha}$ measurements obtained experimentally.

The uncertainty in $\Phi_{\text {wall }}$ consists of the uncertainty of the measured intensity signal $\Delta I_{H_{\alpha}}$ and the uncertainty of the calibration quantities $\Delta c_{H \alpha}$ and $\Delta \alpha_{D E G A S} . \Delta I_{H_{\alpha}}$ is obtained as the standard deviation $\sigma\left(I_{H_{\alpha}}\right)$ of the $I_{H_{\alpha}}$ during the analysis window (a real measurement uncertainty), the calibration error of the $\mathrm{H}_{\alpha}$ photon flux measurement (a systematic uncertainty), and the estimated uncertainty in the DEGAS calculation and scaling procedure $\Delta \alpha_{D E G A S}$, which is a systematic uncertainty assuming the variation in $n_{e}$ and $T_{e}$ is moderate during the analysis time window and in the discharges compared. The uncertainty $\Delta \alpha_{D E G A S}$ is determined by running the DEGAS calculations three times for each magnetic configuration: the first was run using the background temperature and density shown in Fig. 4, the second was run using the values of temperature and density at the lower error bar, and the third was run using the temperature and density at the upper error bar for each configuration. The deviation in the final scale factor between these upper and lower limits of the background plasma is used to determine the uncertainty in the DEGAS calculation and the subsequent scale factor, which is $\sim 16$ percent. This uncertainty, however, again is a systematic uncertainty that arises from the neutral model, and hence it will cancel out for relative comparisons. The total error in the wall source term, determined from linear error propagation, is $\sim 20-25$ percent, but of this total, only $\sim 5$ percent stems from real measurement uncertainties 
which do not cancel in relative comparisons.

The final particle balance is analyzed with the aforementioned terms (i.e. $N_{\text {tot }}, \frac{d N_{\text {tot }}}{d t}, \Phi_{\text {gas }}$, and $\Phi_{\text {wall }}$ ) on a shot-by-shot basis. The terms that vary in Equation 5 (i.e. everything except the fueling efficiency coefficients) are all shown in Fig. 8 as a function of mean line-averaged density (calculated from the central interferometer channel during the analysis window). Data from the EdgeIslands configuration are shown with red circles and data from the No-Edge-Islands configuration are shown with gray diamonds. A single term power scaling was fit to the data and is shown with a solid line for each configuration. The mean standard deviation of the data from the fit line is shown as a shaded region above and below the power law fit line. This overview of the particle balance shows that all terms are comparable, but the time rate change of the density evolution (Fig. 8(b)) as well as the gas fueling flux (Fig. 8(c)) are somewhat different for the No-Edge-Islands case. However, the $\frac{d N_{\text {tot }}}{d t}$ term is roughly one order of magnitude smaller than the $\Phi_{\text {gas }}$ and $\Phi_{\text {wall }}$ terms, and therefore makes only a small contribution to the overall particle balance.

One important element of the analysis is only implicitly visible in Fig. 8: the fueling efficiency $f_{\text {wall }}$ for the recycling and gas flux terms $f_{\text {gas }}$. They are inferred using comparative EMC3-EIRENE modeling as will be discussed in Section VI. This analysis of $f_{\text {eff }}$ aided by EMC3-EIRENE shows a difference in the fueling efficiency of $20 \%$. This difference in the fueling eventually leads to the significant difference in $\tau_{p}$ between both configurations shown in Fig. 8(e). The global particle confinement time $\tau_{p}$ is reduced by up to $25-30 \%$ when the $m / n=8 / 7$ island chain is moved into the plasma. The meaning of this reduction as well as the physics reason for the reduced fueling efficiency which defines this reduction is discussed in Section VII in conjunction with the numerical analysis of the experiments with EMC3EIRENE.

The uncertainty of all the component terms in Equation 5 are then added using linear error propagation to determine the total error for all individual measurements of $\tau_{p}$. This uncertainty is $\sim 14-20$ percent in the EdgeIslands configuration and $\sim 15-18$ percent in the NoEdge-Islands configuration. These quantities can be seen in Fig. 8(e) calculated for each individual measurement. A single term power scale fitting was determined as a function of the plasma density. This fit can be seen in Fig. 8(e).

There are several important pieces of information contained in Fig. 8(e). First, the values of $\tau_{p, H}$ in the EdgeIslands configuration are generally larger than those in the No-Edge-Islands configuration. At a value of $\mathrm{n}_{e}=3.6 \times 10^{18} \mathrm{~m}^{-3}, \tau_{p, H}$ is a factor of 1.32 larger in the Edge-Islands configuration. Additionally, as we observed in the $\tau_{p}^{*}$ measurements, we find a similar trend in the behavior of $\tau_{p, H}$ as a function of the mean $\mathrm{n}_{e}$ : an increasing value of $\tau_{p, H}$ in the Edge-Islands configuration and a decreasing value of $\tau_{p, H}$ in the No-Edge-Islands configuration.

As mentioned above in the discussion of the $\tau_{p}^{*}$ results, these differing trends as a function of plasma density may imply different confinement physics in the Edge-Islands configuration and No-Edge-Islands configuration. We hypothesize that these differing trends are related to the relative ability of undesirable wall-sourced particles to penetrate into the confinement region, as we will discuss in greater detail in Section VII.

\section{Global recycling coefficient in HSX}

If we take the density scaling fits for $\tau_{p, H}$ and $\tau_{p, H}^{*}$ as a function of $\mathrm{n}_{e}$, we can compute $R$ over this same density range. These values, and their respective confidence regions (shown as shaded regions), are plotted in Fig. 9. The confidence regions shown are calculated with linear error propagation and the partial derivative weighting factors contribute strongly to the small total standard deviation. The calculated values of $R$ for the Edge-Islands configuration range from approximately $0.83-0.87$, and the calculated values of $R$ for the No-Edge-Islands configuration range from approximately 0.82-0.78. However, these values lie close to the overlapping range of the error bars, and it can be argued that $R$ is very similar between the two configurations.

\section{EXPERIMENTAL STUDIES OF PARTICLE BEHAVIOR AT W7-X}

In this section, results from a similar assessment of the particle fueling and exhaust balance of both the Increased Iota and Standard configurations at W7-X are shown. This study was performed during the initial operational phase of W7-X, in which the plasma boundary was defined by five poloidal limiters ${ }^{34}$. The device performance exceeded expectations for this initial startup campaign ${ }^{35}$, but diagnostic access and availability was still limited ${ }^{36}$. The main measurements used in this study are the line integrated density $\overline{n_{e}}(t)$ from the single-channel interferometer ${ }^{37}$, Thomson scattering profiles for electron density $n_{e}(r)$ and electron temperature $T_{e}(r)$ and an absolutely calibrated filterscope system $^{38}$, which allowed flexible acquisition of line integrated atomic line emission signals for hydrogen, helium and carbon. The analysis approach was the same as explained before. Effective particle confinement times for helium were measured by perturbative gas injection experiments and the global particle confinement time $\tau_{p}$ was inferred using the same single reservoir particle balance as used for HSX. 


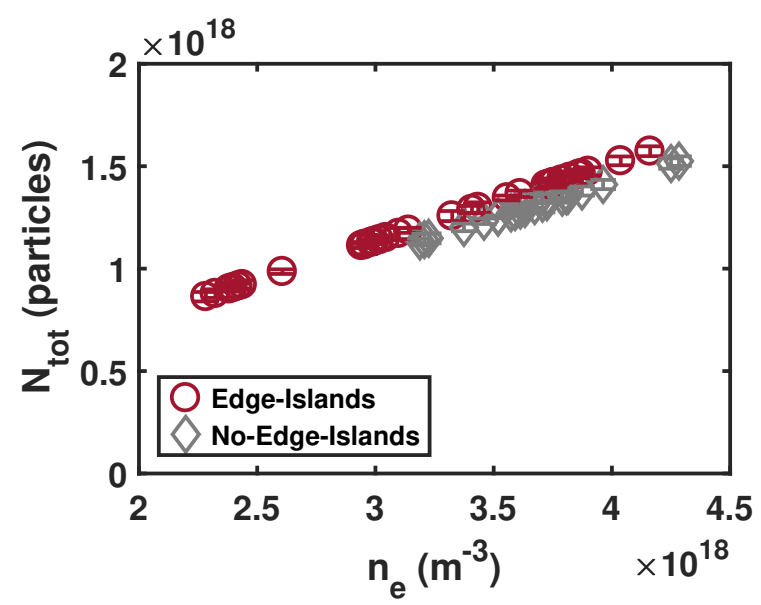

(a)

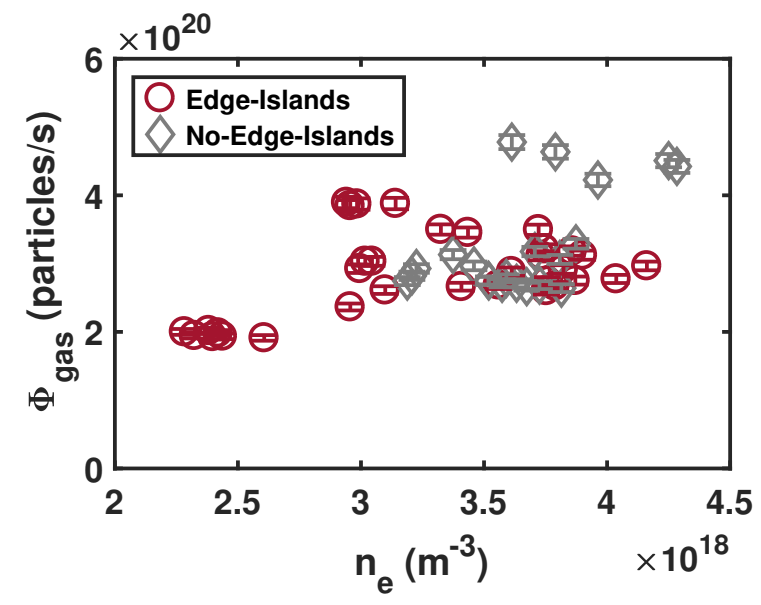

(c)

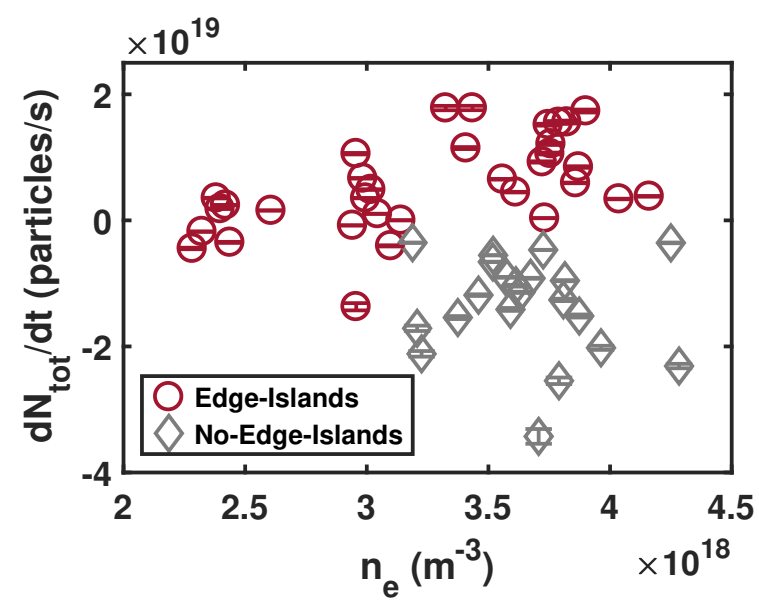

(b)

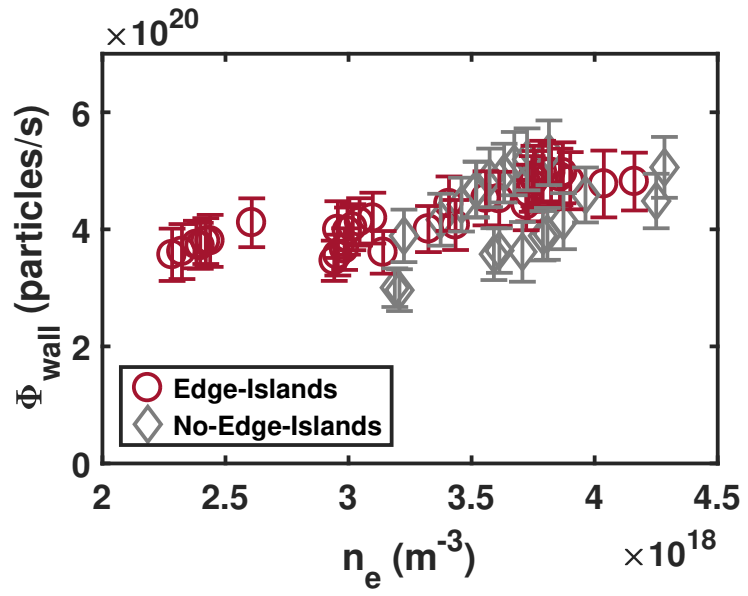

(d)

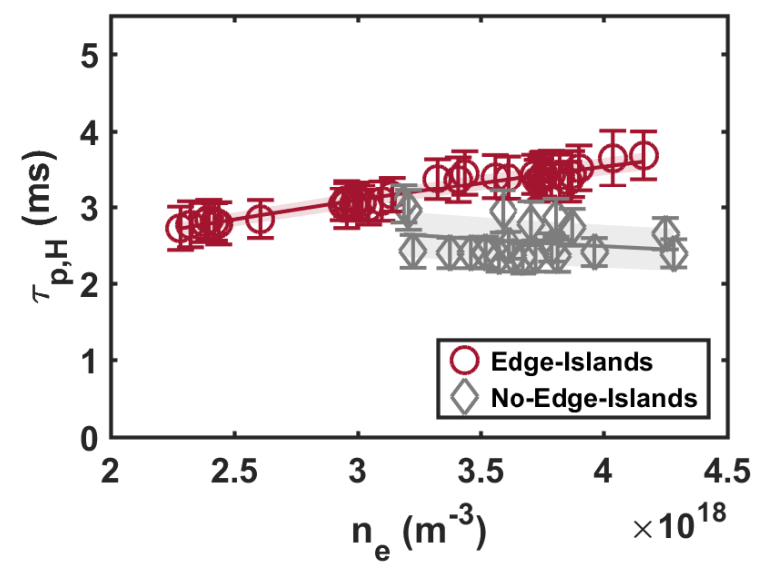

(e)

FIG. 8: Terms in the particle balance analysis plotted as a function of mean line-averaged density measured by the HSX interferometer. Data from the Edge-Islands configuration are shown with red circles and data from the No-Edge-Islands configuration are shown with gray diamonds and gray squares. (a) Values of $\mathrm{N}_{\text {tot }}$, (b) values of $\frac{d N_{\text {tot }}}{d t}$, (c) values of $\Phi_{\text {gas }}$, (d) values of $\Phi_{\text {wall }}$, (e) values of $\tau_{p, H}$. The lines in (e) are single term power function fit lines. The error bars represent the total individual error for each data point, and the shaded regions represent the mean standard deviation between the data points and the fit line. 


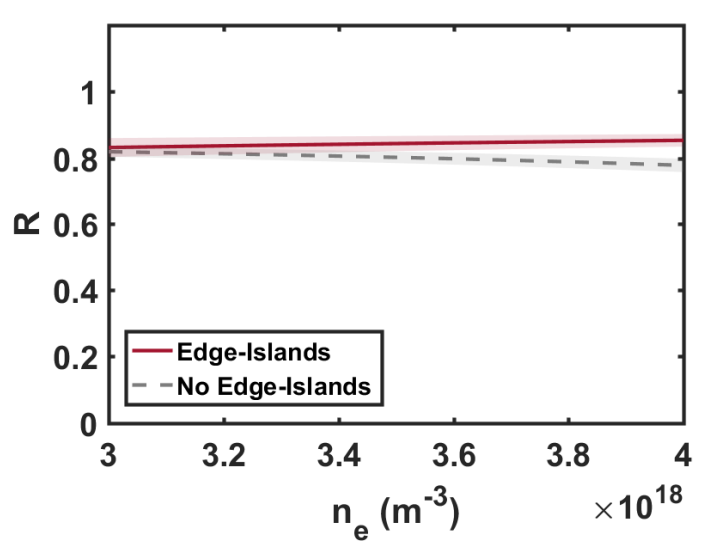

FIG. 9: The global recycling coefficient R for HSX plotted as a function of plasma density. This value is calculated using Equation 2. The shaded regions are the sum in quadrature of the uncertainty in all of the component terms in this analysis. Data from the Edge-Islands configuration are shown with a solid red line and data from the No-Edge-Islands configuration are shown with a dotted gray line.

\section{A. Effective particle confinement time in W7-X}

A fast piezo valve was used to make a single $20 \mathrm{~ms}$ helium injection of $1 \times 10^{19}$ particles into a hydrogen plasma. The number of injected helium particles was kept constant in each discharge. A filterscope diagnostic ${ }^{39-41}$ was used to measure the emission decay of both He-I at $\lambda=667.8 \mathrm{~nm}$ and He-II at $\lambda=468.6 \mathrm{~nm}$ in two separate poloidal positions at the toroidal location of the gas injection in module 5. An additional He-I channel observing the limiter in module three was used to determine He-I decay at this position as well. The details of the particular experimental setup are described $\mathrm{in}^{38}$.

In Fig. 10, an example of He-II line emission time trace is shown, which was used to determine $\tau_{p, \mathrm{He}-\mathrm{II}}^{*}$. Here the He-II line emission is shown and the time of the helium injection is indicated. Shortly after the gas injection, a fast peaking of the helium emission is seen with a rapid immediate decay from direct ionization $(\sim$ several $\mathrm{ms}$ ). The effective confinement time, however, is a result of the propagation of the gas injection through the system where it recycles and is re-ionized. Hence the longer timescale is used (between 400 and $600 \mathrm{~ms}$ ), marked as orange in Fig. 10, to determine $\tau_{p, \mathrm{He}}$. Note that in both cases, the signal used for the decay measurement appears linear on this $\log$ scale plot and is therefore suitable for exponential fitting.

The decay of helium impurity emission was measured to determine $\tau_{p, \text { He-I }}^{*}$ and $\tau_{p, \text { He-II }}^{*}$. These helium impurity injections were performed in both magnetic configurations and also at two levels of heating power: low power (2 MW) and high power (2.7 MW). The characteristic decay time determined from both He-I (viewing limiter

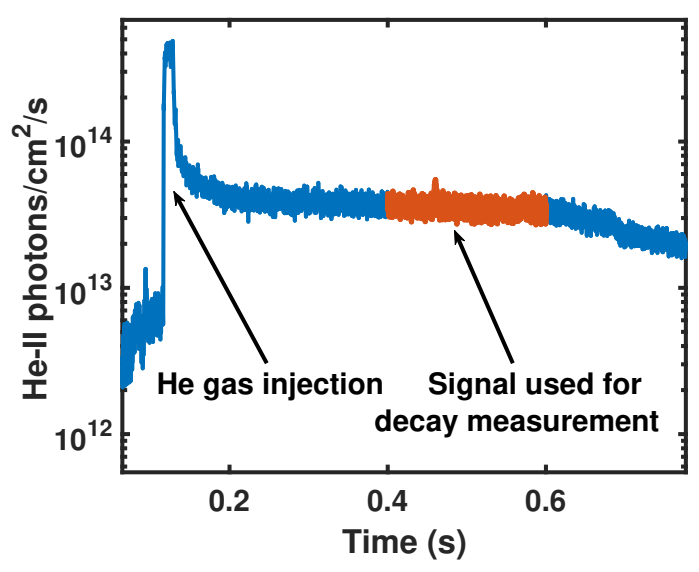

FIG. 10: A representative He-II emission time trace from W7-X showing the helium gas injection at $250 \mathrm{~ms}$ and the signal used to determine $\tau_{p, \mathrm{He}-\mathrm{II}}^{*}$ which is highlighted in orange (400-600 ms). An exponential curve was fit to this section of data and the resulting decay constant yields the value of $\tau_{p, \mathrm{He}-\mathrm{II}}^{*}$.

3) and He-II (at the same toroidal angle as the gas injection) measurements are shown in Fig. 11. The value of $\tau_{p, \mathrm{He}-\mathrm{I}}^{*}$ and $\tau_{p, \mathrm{He}-\mathrm{II}}^{*}$ is always longest in the Increased Iota configuration.

Given that a measurement of $\tau_{p, H}^{*}$ is necessary to compute the global recycling coefficient $\mathrm{R}$, we perform an additional exponential fit on the monotonically rising interferometer density signal. Because the helium injection is relatively small compared to the wall and limiter hydrogen source rates (roughly a factor of 10 smaller, as will be shown in what follows), the interferometer density can be assumed to represent predominately hydrogen. This analysis yields $\tau_{p, H}^{*}$ which is shown in Fig. 11. Because the density profile is monotonically rising, the fit coefficient of the exponential is negative, which in turn gives a negative value of $\tau_{p}^{*} H$, indicating that in this single reservoir balance the fueling term overcomes the outflow $-N / \tau_{p}$. This over-fueled situation will be discussed again in Section $\mathrm{V}(\mathrm{C})$, where the recycling coefficient is extracted.

It should be noted that the error bars in all plots shown in Fig. 11 are the uncertainties in the 95 percent confidence exponential fit for each individual data point. The shaded regions above and below the fit line indicate the mean standard deviation from the fit line for data in each configuration.

\section{B. Particle confinement time in W7-X}

To infer the particle confinement time, $\tau_{p}$, the same particle balance as discussed in Section III is used. The particle balance described in Equation 3 was adapted for $\mathrm{W} 7-\mathrm{X}$ as follows. Because there is no external gas fueling 

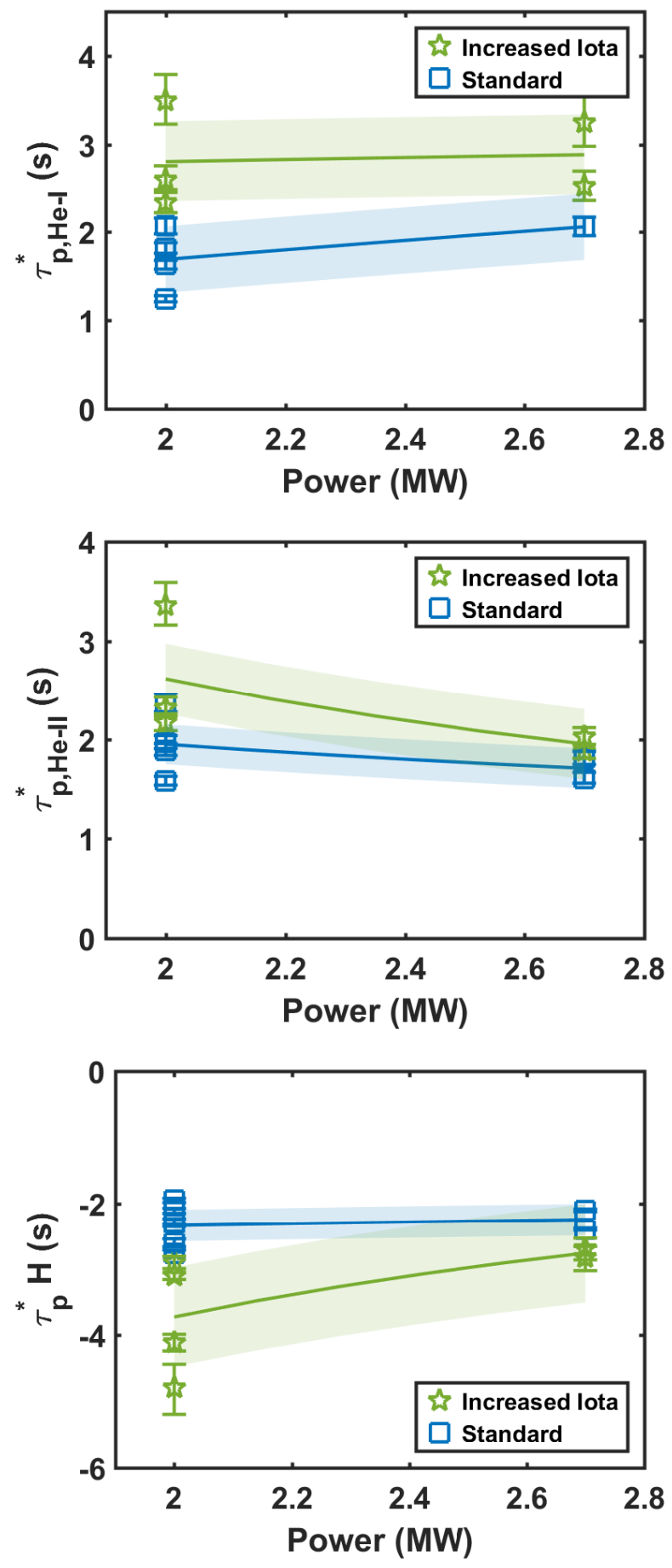

FIG. 11: (Top) $\tau_{p \text {,He-I }}^{*}$ as a function of heating power measured from the decay of a perturbative helium puff using the $468.6 \mathrm{~nm}$ line. The solid lines indicate a power function fit for each configuration. (Middle) $\tau_{p \text {,He-II }}^{*}$ as a function of heating power measured from the decay of a perturbative helium puff using the 667.8 $\mathrm{nm}$ line. The solid lines indicate a power function fit for each configuration. (Bottom) $\tau_{p, H}^{*}$ as a function of

heating power measured from the decay of the interferometer line-averaged density. The values of $\tau_{p, H}^{*}$ are negative because the plasma density is rising rather than falling. The solid lines indicate a power function fit for each configuration. (i.e. no main gas fueling nor perturbative fueling) during the analysis window, this term can be neglected, leaving:

$$
\tau_{p}=\frac{N_{\text {tot }}}{-\frac{d N_{\text {tot }}}{d t}+f_{\text {lim }} \Phi_{\text {lim }}+f_{\text {wall }} \Phi_{\text {wall }}}
$$

Similar to HSX, the $\mathrm{N}_{\text {tot }}$ term was determined by multiplying the line averaged density from the W7-X interferometer by the confinement volume for each configuration computed with EMC3-EIRENE ${ }^{42}$. This process is described by Equation 6. The calculated volume of the W7-X Standard configuration is $27.14 \mathrm{~m}^{3}$ and the volume of the Increased Iota configuration is $26.06 \mathrm{~m}^{3}$. To find $\frac{d N_{\text {tot }}}{d t}$, the change in $\mathrm{N}_{\text {tot }}$ during the analysis window from 400-600 ms was determined. The values of the $\mathrm{N}_{\text {tot }}$ and $\frac{d N_{\text {tot }}}{d t}$ terms for each shot used in the analysis are shown in Fig. 12(a) and (b), respectively. Data in the Increased Iota configuration are shown as green stars and data in the Standard configuration are shown as blue squares.

The uncertainty in the value of $\mathrm{N}_{\text {tot }}$ was determined by first removing the constant rise (since this rise is already accounted for in the $\frac{d N_{\text {tot }}}{d t}$ term) by fitting a line to the data and subsequently subtracting this quantity. Then we find the standard deviation in the interferometer signal during our analysis window. This uncertainty is applied to both the $\mathrm{N}_{\text {tot }}$ and the $\frac{d N_{\text {tot }}}{d t}$ terms and is approximately 1-3 percent. These uncertainties, $\Delta_{N_{t o t}}$ and $\Delta_{\Delta N_{\text {tot }}}$ are displayed as error bars for each data point in Fig. 12(a) and (b).

For W7-X, the limiter particle flux term, $\Phi_{\text {lim }}$ (Equation 10), and the wall particle flux term $\Phi_{\text {wall }}$ (Equation 8), are defined as:

$$
\begin{gathered}
\Phi_{l i m}=c_{H_{\alpha}} I_{H_{\alpha}} A_{l i m} \beta_{S X B} \\
\Phi_{\text {wall }}=c_{H_{\alpha}} I_{H_{\alpha}} A_{\text {wall }} \beta_{S X B}
\end{gathered}
$$

where $c_{H_{\alpha}}$ is an absolute calibration factor, $I_{H_{\alpha}}$ is the intensity signal with units [photons s${ }^{-1} \mathrm{~cm}^{-2}$ ], $A_{\text {lim }}$ is the the surface area of the W7-X limiters, $A_{\text {wall }}$ is the total estimated wall surface area, and $\beta_{S X B}$ is the value of the $S / X B$ coefficient in the relevant range of edge temperatures and densities, were both determined through absolutely calibrated filter-scope measurements ${ }^{38}$.

To determine $\Phi_{\text {lim }}$, the filterscope channel spot size covered the entire width of limiter 3 (approximately 15 $\mathrm{cm}$ in diameter) but only approximately 20 percent of the height, and hence only 20 percent of the surface area. We assume that this spot is large enough to be representative of the general limiter particle flux. This assumption is not ideal, but necessary to complete this analysis. Additionally, because only one limiter had both a filterscope view and calibrated $\mathrm{H}_{\alpha}$ photon flux, it is necessary to assume that all five limiters had identical particle fluxes. The $\mathrm{H}_{\alpha}$ photon flux per area derived from the viewing location on limiter 3 was multiplied by the total limiter area of 0.86 
$\mathrm{m}^{2}$ for all five limiters, determined from design drawings for the limiters, to produce the total photon flux. This total photon flux was multiplied by the $S / X B$ coefficient 13.36 based on limiter Langmuir probe temperature and density data, and this value was multiplied by 2 to include the contribution from molecular particles ${ }^{43}$. This yields the total value of $\Phi_{\text {lim }}$.

The uncertainty in the limiter particle flux, $\Delta \Phi_{\lim }$ is determined through linear error propagation of several terms: 1) the estimated error in the $\mathrm{H}_{\alpha}$ signals due to calibration error $\Delta C_{H_{\alpha}}(\sim 11$ percent $\left.), 2\right)$ the standard deviation of the $\mathrm{H}_{\alpha}$ signals during the analysis window $\Delta I_{H_{\alpha}}(\sim 15-30$ percent $\left.), 3\right)$ the estimated error in the limiter surface area estimation ( $\sim 5$ percent $)$, and 4$)$ the estimated error in the $\mathrm{S} / \mathrm{XB}$ coefficient between 30-60 $\mathrm{eV}(\sim 13$ percent). This error is plotted for each data point in Fig. 12(d). The total value of $\Delta \Phi_{\lim }$ is $\sim 20-40$ percent.

To determine $\Phi_{\text {wall }}$, the measured values of $\mathrm{H}_{\alpha}$ photon flux was used, this time from a line of sight intersecting the W7-X wall. Like the $\Phi_{\text {lim }}$ measurement, the $\Phi_{\text {wall }}$ measurement is based purely on experimental measurements. The surface area of the W7-X wall was estimated by assuming a cylindrical geometry. Using a major radius of $5.5 \mathrm{~m}$ and a minor radius of $0.53 \mathrm{~m}$, this yielded a wall area of $110 \mathrm{~m}^{2}$. Based on camera images captured by the visible fast camera diagnostic ${ }^{44}$, in general only the inboard half of the W7-X wall appeared to radiate. Based on these images, we therefore divide the total wall area in half to yield an effective wall source area of 55 $\mathrm{m}^{2}$ with an estimated 20 percent uncertainty to account for both geometrical and plasma source estimation uncertainties. We multiply the filterscope measured $\mathrm{H}_{\alpha}$ photon flux by the effective wall source area, and then multiply this product by the $\mathrm{S} / \mathrm{XB}$ coefficient 11.38 based on edge measurements of temperature and density ${ }^{45}$. Finally we multiply this quantity by 2 to include the contribution from molecular particles in the same manner as the limiter flux measurements. This yields the total value of $\Phi_{\text {wall }}$.

The uncertainty in the wall particle flux $\Delta \Phi_{\text {wall }}$ is determined in a similar manner to the limiter particle flux. It is determined through linear error propagation of the following terms: 1) the estimated error in the $\mathrm{H}_{\alpha}$ signals due to calibration error $\Delta C_{H_{\alpha}}(\sim 11$ percent $\left.), 2\right)$ the standard deviation of the $\mathrm{H}_{\alpha}$ signals during the analysis window $\Delta I_{H_{\alpha}}(\sim 10-15$ percent $\left.), 3\right)$ the estimated error in the wall surface area estimation $(\sim 20$ percent $)$, and the estimated error in the $\mathrm{S} / \mathrm{XB}$ coefficient between 30 $40 \mathrm{eV}$ ( $\sim 7$ percent). This error is plotted for each data point in Fig. 12(c). The total value of $\Delta \Phi_{\text {wall }}$ is $\sim 20-30$ percent.

Now that all values necessary for the particle balance have been obtained (with the exception of the fueling efficiency coefficients, which will be discussed in Section VI), the final value of $\tau_{p, H}$ can be determined. This value is plotted as function of input heating power in Fig. 12(e). Over the range of heating power, the values of $\tau_{p, H}$ are approximately between $150 \mathrm{~ms}$ and $200 \mathrm{~ms}$, with $\tau_{p}$ decreasing with increasing heating power. Similar to HSX, a single-term power series fitting was used to fit the data. This fit is shown as a solid line in green and blue for the Increased Iota and Standard configurations, respectively. At a heating power of $2.35 \mathrm{MW}$, the value of $\tau_{p, H}$ from the fit lines is approximately a factor of 1.05 larger in the Increased Iota configuration relative to the Standard configuration.

The uncertainty of all the component terms in Equation 9 are then determined through linear error propagation to determine the total error for all individual measurements of $\tau_{p}$. This uncertainty is $\sim 21$ percent in the Increased Iota configuration and $\sim 22$ percent in the Standard configuration. These quantities can be seen in Fig. 12(e) calculated for each individual measurement. Similar to HSX, a power scaling fitting was determined, now as a function of heating power rather than density. This fit can be seen in Fig. 12(e). The shaded region indicates the mean standard deviation of the data points from the fit line. This is meant to give a confidence region for the power scale fit.

\section{Global recycling coefficient in W7-X}

With both $\tau_{p}^{*}$ and $\tau_{p}$ extracted from measurements at W7-X, Equation 2 is used to determine the global recycling coefficient $R$. Since the measurements of both $\tau_{p}^{*}$ and $\tau_{p}$ were obtained as a function of heating power, $R$ is computed over this same range using the power scaling fit lines which were previously determined. The values we obtain are shown in Fig. 13. The shaded confidence regions shown are calculated using linear error propagation and the partial derivative weighting factors contribute strongly to the small total standard deviation. This calculation yields a value of $R$ approximately equal to 1.1 for both levels of heating power. It should be noted that the value of $R$ computed in each configuration is slightly different but agree within error bars.

This value of $R \approx 1.1$ represents particle release from the wall, i.e. a situation of over-fueling the plasma by wall particle release. As a consequence, the line-averaged density as measured by the interferometer was seen to rise monotonically in all plasmas in this study despite no additional external gas input. This density rise is a result of particle release by the limiter and/or the main wall during the analysis window. This over-fueling situation is consistent with minimal wall conditioning performed before the startup campaign experiments were conducted (e.g. baking at 150 degrees $\mathrm{C}$ only, which is below the threshold to remove impurities from carbon ${ }^{46}$ ). This result provides some additional confidence in our measurements of $\tau_{p}^{*}$ and $\tau_{p}$ since the $R$ they yield is consistent with plasma observations and the anticipated wall conditions during the startup campaign at W7-X. 


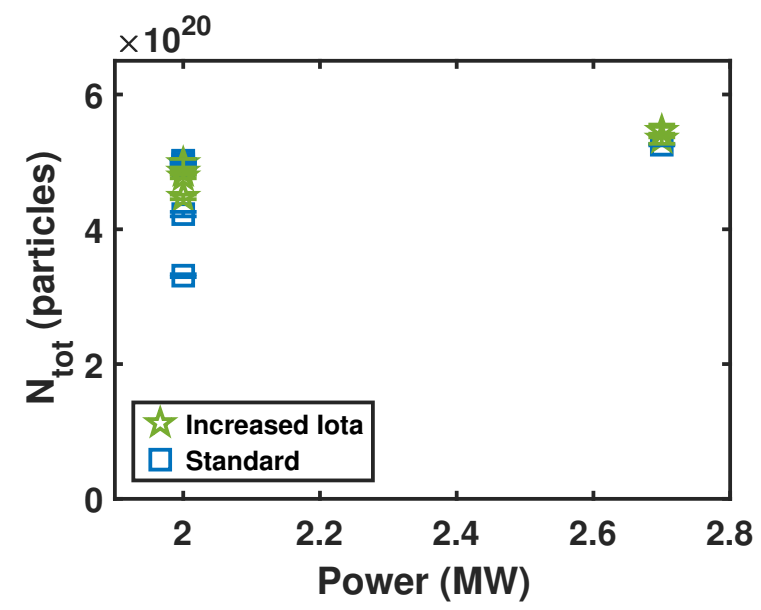

(a)

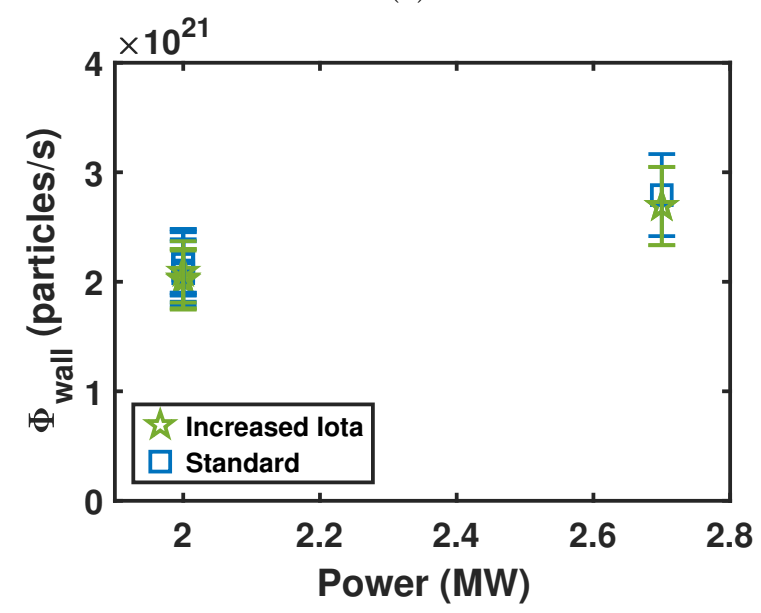

(c)

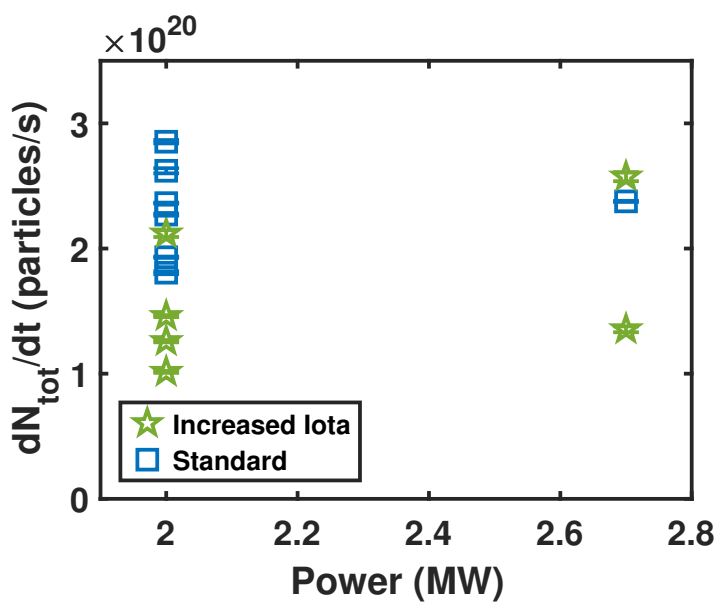

(b)

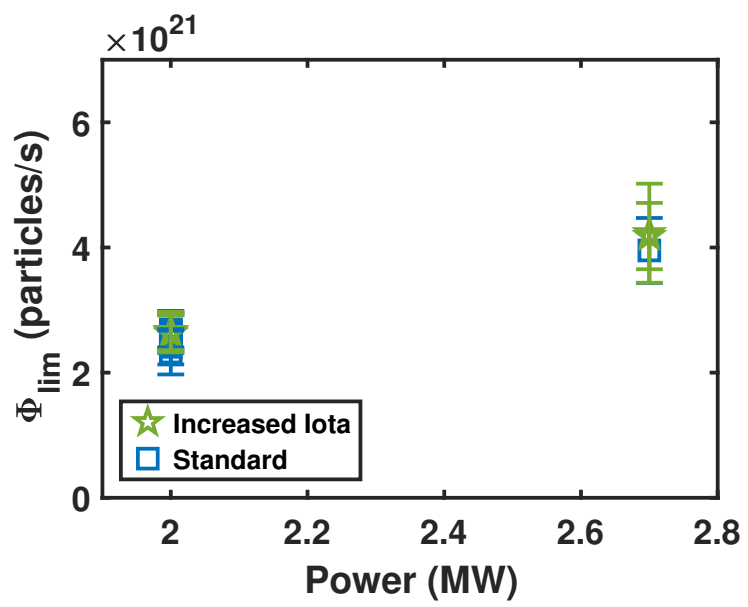

(d)

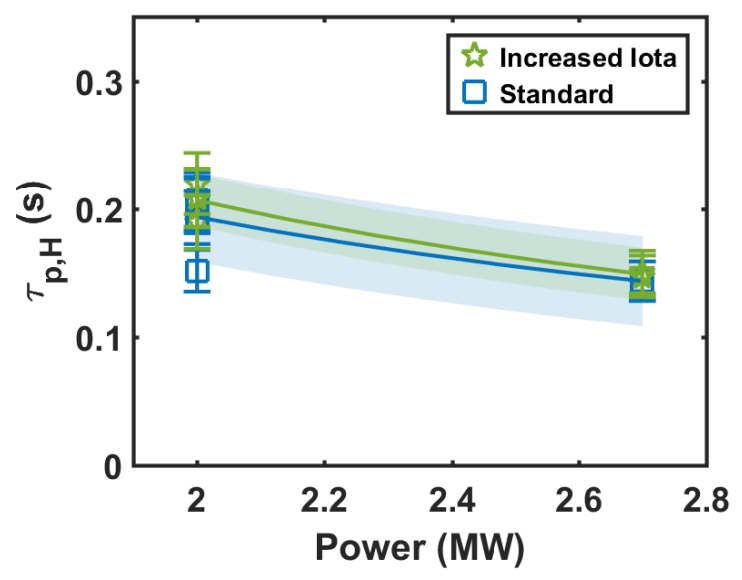

(e)

FIG. 12: Terms in the W7-X particle balance analysis for each individual plasma shot as a function of input power. Data from the Increased Iota configuration are shown with green stars and data from the Standard configuration are shown with blue squares. (a) Values of $\mathrm{N}_{\text {tot }}$, (b) values of $\frac{d N_{\text {tot }}}{d t}$, (c) values of $\Phi_{\text {wall }}$, (d) values of $\Phi_{\text {lim }}$, (c) values of $\tau_{p, H}$. A power series fit to $\tau_{p, H}$ for each configuration is shown with a corresponding green and blue solid line. The power series fitting parameters are printed on the plot. The shaded region indicates the mean standard deviation of the data points from the fit line. 


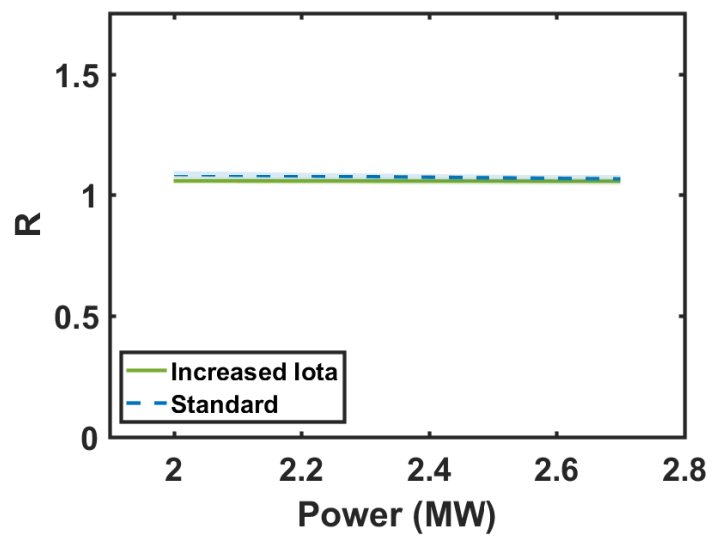

FIG. 13: The global recycling coefficient $\mathrm{R}$ for $\mathrm{W} 7-\mathrm{X}$ plotted as a function of heating power. This value is calculated using Equation 3. The shaded regions are the sum in quadrature of the uncertainty in all of the component terms in this analysis. Data from the

Standard configuration are shown with a solid blue line and data from the Increased Iota configuration are shown with a dotted green line.

\section{EMC3-EIRENE SIMULATIONS OF PARTICLE PENETRATION IN HSX AND W7-X}

A key quantity in the particle balance analysis is the fueling efficiency, $f_{\text {eff }}$, described in Equation 4. However, making this measurement is experimentally challenging. There is no direct measurement of the neutral particle profile available at either HSX nor W7-X, and hence the neutral/ion ratio across the radius would have to be reconstructed using proxy quantities like the Balmer- $\alpha$ emission combined with plasma density and temperature profiles, which may or may not be known.

Instead, EMC3-EIRENE was used to simulate all four magnetic configurations examined in this work ${ }^{42,47}$. Note that the values of $T_{e}$ and $n_{e}$ are self-consistently calculated based on the prescribed input power and core density of each case. The simulation parameters between the two pairs of configurations were kept as similar as possible to facilitate comparison between the cases. These choices were informed by Thomson profile measurements of plasma density and temperature like those shown in Figs. 4 and5.

For HSX simulations, only wall-sourced neutral particles were included in the EMC3-EIRENE simulation (i.e. no gas injection was simulated) and for the W7-X simulations, only limiter-sourced particles were included in the EMC3-EIRENE simulation (i.e. no wall-sourced particles were simulated), because these were expected to be the dominant particle source in each respective device. The fueling efficiency was then determined by separating the grid into regions of open and closed field lines ${ }^{48}$ based on a user-supplied value of threshold $\mathrm{L}_{c}$. In general only the value of $f_{\text {eff }}$ for the plasma confinement region was considered because this quantity was being used for a single-reservoir particle balance, as described in Section III.

Calculations of the fueling efficiency in HSX are shown in Fig. 15 as a function of the total number of plasma particles in the simulation domain, $\mathrm{N}_{t o t}$. (The value of $\mathrm{N}_{\text {tot }}$ may appear small since the EMC3-EIRENE grid does not include the whole HSX volume. The currently-attainable density is circled on this plot; the rest of the points are projections to higher densities that cannot be experimentally tested at HSX. At this realistic density value, the fueling efficiency in the Edge-Islands configuration is 0.70 and the fueling efficiency in the No-Edge-Islands configuration is 0.87 , a factor of 1.24 increase. This increase is well outside of the estimated errors in the $f_{\text {eff }}$ calculations (5-7 percent).

\section{A. Fueling efficiency for HSX}

The particle source distribution showing the penetration of the ionized particle source in the HSX EdgeIslands and No-Edge-Islands configurations is shown in Fig. 14. In this figure, the two top plots show the plasma temperature profiles modeled by EMC3-EIRENE and they highlight the edge island geometry (or lack thereof) in both configurations. In the two bottom plots, the ionization source rate $S_{p}$ (in $A$, or particles/s) in each configuration is shown.

In Fig. 14, the effect of the edge magnetic structure becomes clear: in the Edge-Islands configuration, the ionization region is substantially more localized in the upper part of the bean shaped cross-section and the particle penetration length is shorter as compared to the No-Edge-Islands configuration. The scale of the $\mathrm{S}_{p}$ plots is adjusted (i.e. auto-scaled) to show this difference more clearly between both configurations. The region in which more of the neutral source rate is produced is the X-point region in between the island tips at the upper part of this bean shaped cross-section. Because of this reduced neutral penetration length into the plasma, the overall fueling efficiency is reduced in the Edge-Islands configuration. This reduction can be seen in Fig. 15 for both realistic HSX plasma parameters and also in the characteristic projected for higher plasma densities by EMC3EIRENE modeling.

We postulate that the $8 / 7$ island structure, because it is located within the ionization source region, is able to decouple the source location from the confinement region. This decoupling is primarily due to the locally increased temperatures at the $8 / 7$ island $\mathrm{X}$-point which result in a larger ionization percentage outside the LCFS. Note that in the Edge-Islands configuration, the ionization region is smaller and peaks near the $8 / 7$ island $\mathrm{X}$-point. This is in contrast to the plasma source rate in the No-EdgeIslands case which is more diffuse and penetrates more deeply into the confinement region.

Flux-surface averaging these EMC3-EIRENE data 


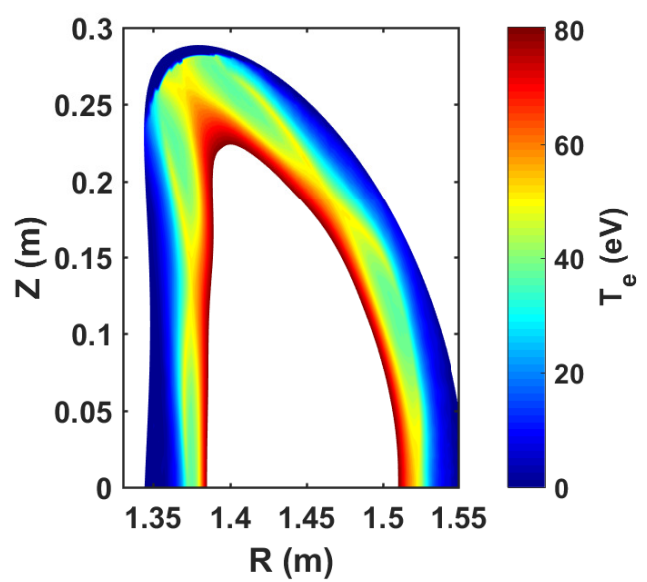

(a)

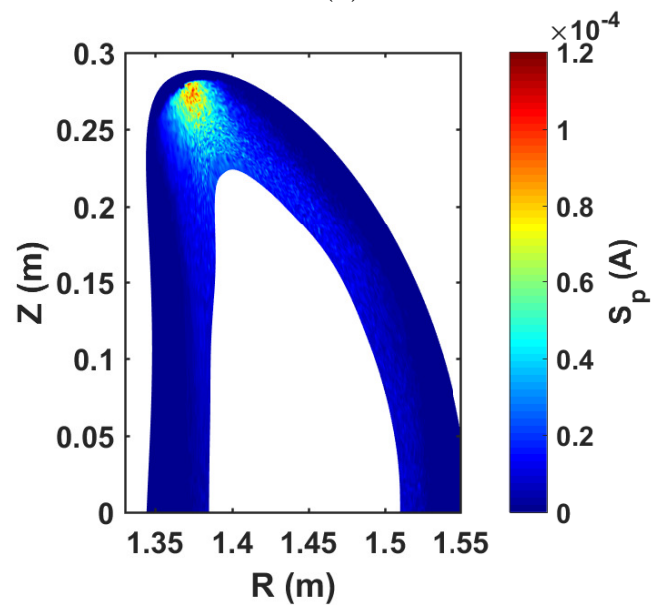

(c)

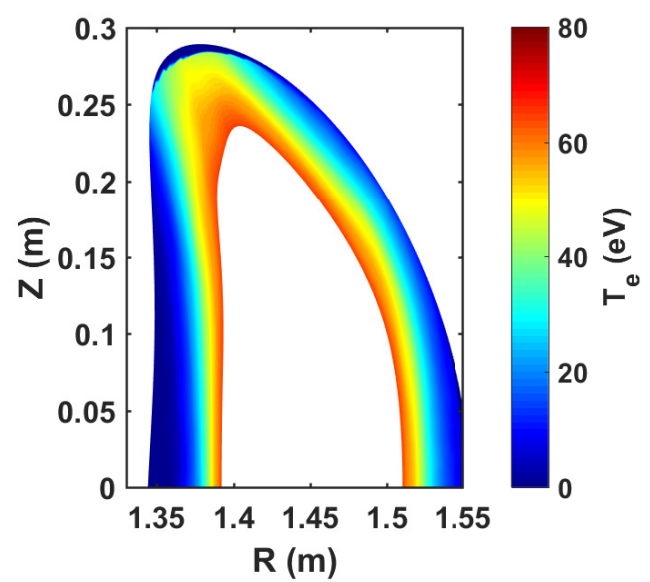

(b)

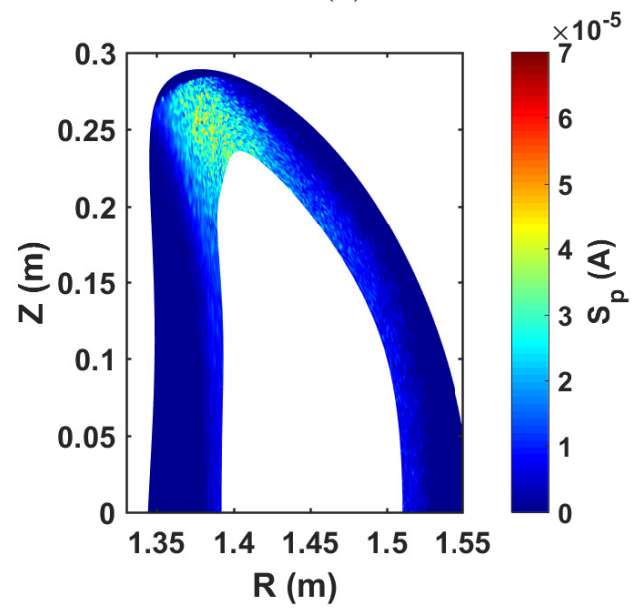

(d)

FIG. 14: Figure showing the relationship between the edge plasma temperature, the magnetic topology, and the ionization region. (Top) Self-consistently calculated EMC3-EIRENE background plasma temperature in the Edge-Islands configuration (a) and the No-Edge-Islands configuration (b). In the Edge-Islands configuration, the $8 / 7$ island structure can clearly be seen in the temperature plot. (Bottom) EMC3-EIRENE plots of the plasma source

rate, $\mathrm{S}_{p}$, in the Edge-Islands configuration (c) and the No-Edge-Islands configuration (d). Note that in the

Edge-Islands configuration, the ionization region is smaller and peaks near the $8 / 7$ island $\mathrm{X}$-point. This is in contrast to the source rate in the No-Edge-Islands case which is more diffuse and penetrates more deeply into the confinement region.

produce more quantitative results of how the ionization domain is affected in each of these configurations. The flux surface average was performed by determining the effective radius of each EMC3-EIRENE grid cell in terms of the known magnetic axis and 3-D last closed flux surface of each magnetic configuration, determined through field line following. All grid cells within the same effective radius bin were then accordingly flux-surface averaged. The results of this calculation are shown in Fig. 16.

Figure 16 clearly illustrates how the edge magnetic geometry affects the flux surface averaged ionization source rate in HSX. In the No-Edge-Islands configuration the ionization source profile is peaked at approximately $\mathrm{r} / \mathrm{a}=0.95$, with a substantial ionization rate both inside and outside the LCFS. In contrast, in the Edge-Islands configuration, the ionization rate is peaked outside the LCFS at approximately $\mathrm{r} / \mathrm{a}=1.05$, with a larger fraction of ionizations taking place outside, rather than inside, the LCFS. These results demonstrate that both the fueling efficiency and ionization domain itself are impacted by the insertion or removal of the $8 / 7$ island in the HSX edge. 


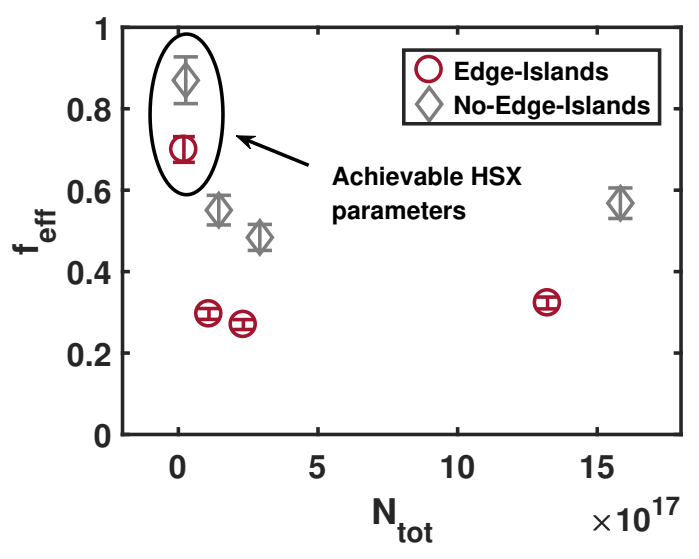

FIG. 15: Figure showing EMC3-EIRENE calculated fueling efficiency, $f_{\text {eff }}$ for both the Edge-Islands (red circles) and No-Edge-Islands (gray diamonds) configurations for a density scan. Values of $\mathrm{f}_{\text {eff }}$ determined for realistic HSX parameters are noted on the plot. $f_{\text {eff }}$ is plotted as a function of $\mathrm{N}_{\text {tot }}$, the total number of particles in the simulation.

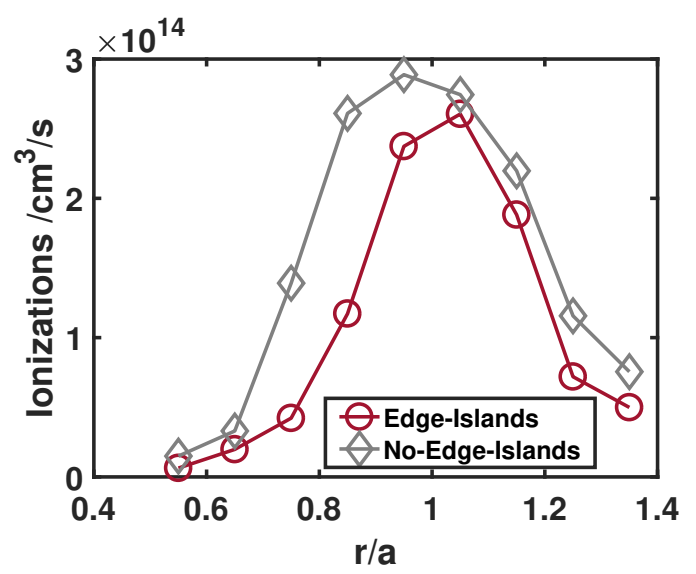

FIG. 16: Plot of EMC3-EIRENE flux surface averaged ionization source rate for hydrogen in both the

Edge-Islands and No-Edge-Islands configurations in HSX. In the Edge-Islands configuration, the flux surface averaged ionization source rate peaks at approximately $\mathrm{r} / \mathrm{a}=1.05$, outside the LCFS. In the No-Edge-Islands configuration, the flux surface averaged ionization source rate peaks at approximately $\mathrm{r} / \mathrm{a}=0.95$, inside the LCFS.

\section{B. Fueling efficiency for W7-X}

Figs. 17 and 18 show a plot of the ionization source rate $S_{p}$ calculated in W7-X, the former in real space coordinates and the latter in flux surface averaged coordinates. The ionization source rate is key to determining the fueling efficiency (see Equation 4), as the quantity $f_{\text {eff }}$ is defined as the total number of ionizations that occur inside the confinement region divided by the total number of

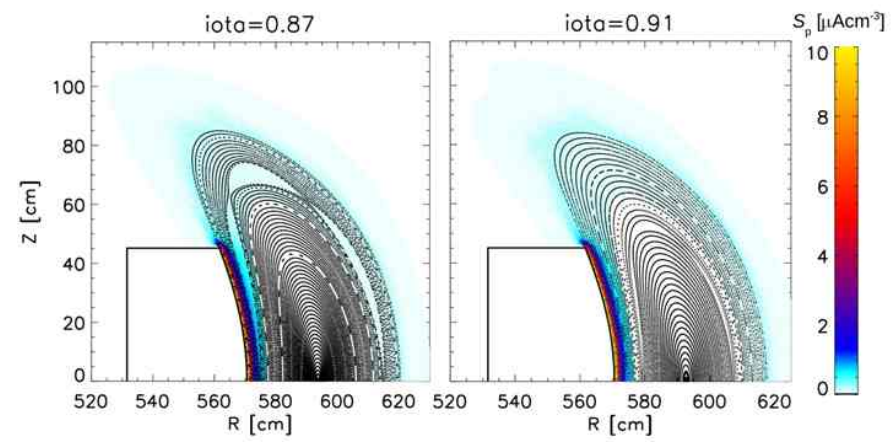

FIG. 17: EMC3-EIRENE calculation of ionization source rate, $\mathrm{S}_{p}$. Despite the change in edge structure, there were no substantial changes in the ionization source distribution. A flux surface averaged plot of these data is shown in Fig. 18.

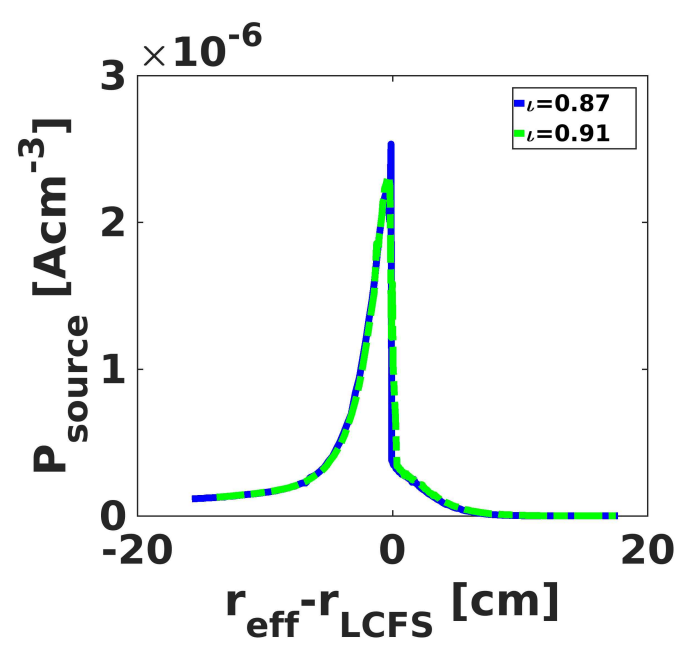

FIG. 18: Plot of EMC3-EIRENE flux surface averaged ionization source rate for helium in both the Standard configuration (blue solid line) and Increased Iota configuration (green dotted line). The helium source profile is very similar to the hydrogen source profile

(not pictured). Plot courtesy F. Effenberg.

ionizations. Data in the Increased Iota configuration are shown with a green dotted line and data in the Standard configuration are shown with a blue solid line. This plot shows very little difference in the limiter particle source rate profile between the Increased Iota and the Standard configurations. This lack of difference in source rate profile, despite moving the $5 / 5$ and $5 / 6$ island chains, results in very little difference in the calculated fueling efficiency coefficients.

Fig. 19 shows the fueling efficiencies for the Increased Iota configuration and the Standard configuration in W7X plotted as a function of total plasma particles, $N_{\text {tot }}$. Data in the Increased Iota configuration are shown with green stars and data in the Standard configuration are shown with blue squares. Data in this plot are shown 


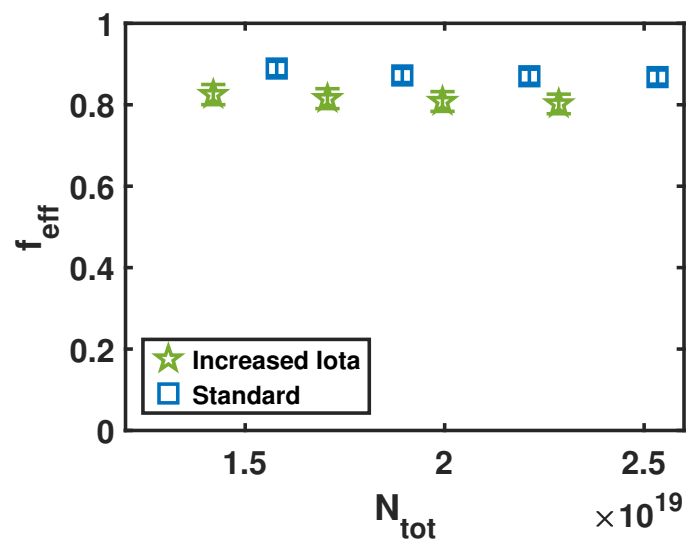

FIG. 19: EMC3-EIRENE calculated numerical values of $f_{\text {eff }}$ for the Standard and Increased Iota configurations in W7-X. The numerical $f_{\text {eff }}$ is shown as a function of total number of plasma particles $N_{\text {tot }}$. Unlike the fueling efficiency study for HSX, this density range was experimentally achieved during the initial operational phase of W7-X.

over the range of achievable densities for W7-X during the limiter operational phase (OP 1.1). This plot shows that in the Increased Iota configuration, the value of $f_{\text {eff }}$ is approximately 0.81 and in the Standard configuration, the value of $f_{\text {eff }}$ is approximately 0.87 . At $\mathrm{W} 7-\mathrm{X}$ this $\sim$ 7 percent difference in particle penetration between the two configurations is less substantial than that observed at HSX between the two configurations ( $\sim 24$ percent). This could be for several reasons, the most likely of which is the close proximity of the limiter to the LCFS. The second is that the higher W7-X plasma densities result in shorter particle ionization lengths, which in turn may cause the ionization region to depend less heavily on the edge topology. Future work will be required to better understand these differences. The uncertainty in the fueling efficiency in the Increased Iota configuration is 3 percent and the uncertainty in the Standard configuration is $\sim 2$ percent, which have been included in the total error in the particle confinement time.

\section{Closure of particle balance using the $f_{e f f}$ inferred}

These values of $f_{\text {eff }}$ extracted from dedicated EMC3EIRENE modeling results were used to close the particle balance and obtain $\tau_{p}$ values. For HSX, these values were inserted directly into Equation 5 for $f_{\text {wall }}$ since the EMC3-EIRENE simulations included only wallsourced neutral particles. For W7-X, these values were inserted directly into Equation 9 for $f_{\text {lim }}$ since the EMC3EIRENE simulations included only limiter-sourced neutral particles.

The remaining terms, $f_{\text {gas }}$ for HSX and $f_{\text {wall }}$ for W7$\mathrm{X}$, however, were adjusted by a constant factor to bring these penetration fractions closer to values consistent with the difference in edge geometry. These quantities could not be directly simulated in EMC3-EIRENE or inferred experimentally. A scaling of the EMC3-EIRENE $f_{\text {eff }}$ prediction was used, rather than a slab calculation for example, because EMC3-EIRENE fully accounts for the 3-D magnetic structure. This scaling was done in the following way:

For the HSX value of $f_{\text {gas }}$, the EMC3-EIRENE prediction of $f_{\text {eff }}$ was reduced by a factor of two in each configuration to yield values of 0.35 and 0.44 for the EdgeIslands and No-Edge-Islands configuration, respectively. This factor of 2 was used to reduce the values of $f_{\text {eff }}$ calculated for puff-fueling to values closer to that found by a previous study at HSX with the DEGAS code $^{49}$ where the gas puff geometry was modeled and $f_{\text {gas }}$ was shown to be $\sim 43$ percent. This reduction makes sense in terms of edge geometry and the position of the gas puff relative to the confinement region. Since gas-sourced particles must, on average, travel a longer distance to the confinement region than those borne at the strike points, it is reasonable that $f_{\text {gas }}$ is smaller than $f_{\text {wall }}$.

For the $\mathrm{W} 7-\mathrm{X}$ value of $f_{\text {wall }}$, the EMC3-EIRENE prediction of $f_{\text {eff }}$ was reduced by a factor of five in each configuration to yield values of 0.19 and 0.17 in the Increased Iota and Standard configurations, respectively. This reduction factor was chosen based on the results of a 1-D slab calculation in which a Maxwell-Boltzmann distribution of $0.026 \mathrm{eV}$ neutral molecular hydrogen penetrating into a background plasma of $40 \mathrm{eV}$ and background density of $1.3 \times 10^{18} \mathrm{~m}^{3}$. These values were chosen based on W7-X edge measurements ${ }^{45}$ and based on the assumption that the main wall is approximately $10 \mathrm{~cm}$ from the confinement region. At a temperature of $40 \mathrm{eV}$, based on atomic cross section data, a molecular hydrogen particle is 8.3 times more likely to ionize than dissociate. If we assume that all particles will either ionize or dissociate (a reasonable assumption in the W7-X edge), this means that 86 percent of particles will ionize and 14 percent will dissociate. Then, however, it is assumed that all dissociated particles ionize within the confinement region due to their increased Franck-Condon energy in the range of several $\mathrm{eV}$. Of the remaining 86 percent of molecular particles, 3 percent of these particles (the high energy tail population of the Maxwell-Boltzmann distribution) are able to penetrate $10 \mathrm{~cm}$ to ionize within the confinement region. This yields a total penetration rate of approximately 17 percent of particles, approximately a factor of five less than the values of $f_{\text {lim }}$ calculated by EMC3-EIRENE. This value is also in improved agreement with previous values of wall-particle penetration in the literature $\mathrm{e}^{22,24,50}$.

\section{DISCUSSION AND SUMMARY}

The systematic analysis of the particle balance on both HSX and W7-X has shown that the edge magnetic struc- 
ture can influence the global particle fueling and exhaust properties. This is an important finding for stellarators, and particularly for the island divertor concept, because in an island divertor the magnetic structure is used to establish a decoupling of the plasma facing components from the main plasma. We demonstrate in this paper with results from HSX that the location and shape of a magnetic island chain in the plasma edge can be used to influence the fueling of recycled particles to the plasma, which is a key factor in plasma density control. Additionally, the edge magnetic topology is shown to have a direct effect on the impurity helium effective confinement time. The efficient exhaust of helium as the fusion born impurity is another central functional goal of a divertor and the results from experiment and modeling presented here support that the edge magnetic structure can be used to affect and potentially optimize helium exhaust. In this section, the central experimental and numerical findings are discussed along these two central findings.

\section{A. Edge topology directly defines particle fueling and exhaust through the ionization source distribution}

One of the key results from this work is that in HSX, the $8 / 7$ magnetic island geometry reduces the penetration fraction of neutral particles into the confinement region (see Fig. 14). The key physics mechanism behind this result is the following. Particle flux exits the LCFS predominately through regions of high curvature. The majority of plasma is then carried through parallel transport to specific localized areas of the wall ${ }^{11}$. When some fraction of these particles recycle, they re-emerge as neutrals in what is effectively the private flux region of the X-point geometry created by the $8 / 7$ island and its intersection with the HSX vacuum vessel (see Fig. 1). As a result, if these particles are ionized within this private flux region, they will be immediately directed back to the wall by outward parallel transport, and the cycle will continue. The $8 / 7$ island geometry therefore helps prevent the recycling particles from ionizing within the confinement region and results in a decreased fueling efficiency as shown by EMC3-EIRENE calculations.

These findings are the result of EMC3-EIRENE simulations in both the Edge-Islands and the No-Edge-Islands configurations. The effect of islands preventing inward flux of neutrals can be clearly seen in Fig. 16. Divertors are designed to prevent this direct penetration of neutral particles $^{5}$, so perhaps it is not surprising that the more "divertor-like" Edge-Islands configuration exhibits positive neutral screening attributes and consequently improved hydrogen (and possibly helium) particle confinement times relative to its No-Edge-Islands counterpart.

This increased plasma temperature at the $8 / 7$ island $\mathrm{X}$ point is not only an EMC3-EIRENE prediction, but has also been experimentally measured using a 2-D movable Langmuir probe in the HSX edge ${ }^{11}$. Temperatures at the X-point were measured to be approximately $60 \mathrm{eV}$ which is well above the minimum ionization potential for neutral hydrogen $(\sim 13 \mathrm{eV})$. Such profiles have not yet been measured for the No-Edge-Islands configuration and could constitute future work.

At W7-X, the radial shift of the magnetic islands in between the Increased Iota and Standard configurations examined in this work does not alter the plasma edge profiles in a similar systematic way. Accordingly, no such clear relation between the changes in the magnetic structure and plasma fueling and exhaust was found. In the Standard configuration, the 5/6 island chain was located several $\mathrm{cm}$ inside the LCFS. However, EMC3-EIRENE modeling shows that the ionization domain in front of the limiter - which defines the LCFS - is very thin as shown in Fig. 17. Hence, the $5 / 6$ island does not directly affect particle fueling into the plasma core domain. In the Increased Iota configuration, the $5 / 6$ island was moved further inward and additionally the $5 / 5$ island was moved into far SOL of W7-X. At this radial location, temperatures and densities are low and hence the island itself does not significantly impact the particle balance.

A summary of the four magnetic configurations explored in this work is shown in Fig. 1. This figure visualizes the relationship between the magnetic island chain(s) and the EMC3-EIRENE flux surface averaged ionization domain in each configuration. The Edge-Islands and NoEdge-Islands configurations at HSX are pictured in (a) and (b), respectively. The Increased Iota configuration and the Standard configuration are pictured in (c) and (d), respectively. In W7-X, essentially no difference was observed in the ionization domain as a result of shifting the $5 / 6$ island inward and introducing the $5 / 5$. However at HSX, where fine adjustments to the magnetic configuration were possible, clear changes to the ionization were observed, namely the radially-outward shifting of the ionization front so that a larger fraction of ionizations took place outside the LCFS. This reduced fueling efficiency in the Edge-Islands configuration ultimately drove the increases in the global particle confinement time calculated using Equation 5 and seen in Fig. 8(e).

\section{B. The positive or negative trend of $\tau_{p}$ depends on the wall-sourced particle contribution}

It is important to note that at HSX the hydrogen particle confinement time scales differently with density in the Edge-Islands configuration when compared to the No-Edge-Islands configuration. The trend in $\tau_{p, H}$ as a function of plasma density is positive in the Edge-Islands configuration while it was found to be negative in the No-Edge-Islands configuration. A similar trend was also seen for at TEXT and several other experiments ${ }^{51-53}$.

At TEXT, simultaneous measurements of the ratio of the limiter particle flux to the wall particle flux provide additional insight into the nature of this rollover. They illustrate that when the ratio of the limiter to wall flux reaches a critical value, in this case $\sim 1.5$, the positive 
trend of particle confinement time with density transitions to a negative trend. This conclusion from TEXT applied to the results from HSX supports the argument that the reduced wall particle contribution in the EdgeIslands configuration contributes to the positive scaling in the value of $\tau_{p, H}$ with density, and likewise the increased wall particle contributed in the No-Edge-Islands configuration contributes to the negative scaling in the value of $\tau_{p, H}$ with density. Without a divertor or some additional magnetic structure to prevent the wall-sourced neutrals from entering the plasma, the increased rates of uncontrollable particle recycling tend to drive down the particle confinement time. This shows the fundamental impact that edge islands can have on particle fueling in stellarator.

\section{Effective helium confinement times increase at HSX and W7-X with magnetic islands in the plasma edge region}

Measurements of the decay of a perturbative helium puff at both HSX and W7-X yielded longer values of $\tau_{p, H e}^{*}$ for the configuration with a magnetic island outside of the last closed flux surface for HSX and for W7-X in the far SOL. At HSX, the value of $\tau_{p \text {,He-II }}^{*}$ was increased by a factor of 1.6 in the Edge-Islands configuration relative to the No-Edge-Islands configuration. At W7-X, the value of $\tau_{p, \text { He-II }}^{*}$ was increased by a factor of 1.2 in the Increased Iota configuration relative to the Standard configuration. Additionally at W7-X, the value of $\tau_{p \text {,He-I }}^{*}$ was increased by a factor of 1.5 in the Increased Iota configuration. These measurements indicate that the edge topology changes at both HSX and W7-X clearly resulted in a reduction of the effective helium confinement time $\tau_{p, H e}^{*}$ when the magnetic islands were not present in the scrape-off layer region. This trend of $\tau_{p, H e}^{*}$ agrees with the trend for $\tau_{p, H}$ at HSX. At W7-X, there was no change measured in $\tau_{p, H}$ despite a significant increase in $\tau_{p, H e}^{*}$ seen with the $5 / 5$ island in the far SOL.

To better understand these seemingly contradictory results, it is important to consider the helium injection location. The helium gas inlet at W7-X was located such that the $\mathrm{He}$ is injected directly into the 5/5 island channel for the Increased Iota configuration. The plasma parameters predicted by EMC3-EIRENE for inside of this island are $T_{e} \approx 4-6 \mathrm{eV}$ and $n_{e} \approx 1.0 \times 10^{18}$ $\mathrm{m}^{-3}$ which result in radial ionization scale length $\sim 5$ $10 \mathrm{~cm}$ for thermal He. This suggests that some amount of the helium injected could be trapped inside of the island. Some experimental evidence which supports this hypothesis are measurements of He-I and He-II emission during and after ECRH turnoff, which suggest that a larger number of helium particles are confined in the Increased Iota configuration. (Helium recombination plays a dominant role below $10 \mathrm{eV}$ where the helium ionization rate coefficient falls off steeply ${ }^{54}$.) These findings suggest that the $5 / 5$ island chain may contribute to this additional helium confinement. Future work with dedi- cated 2-D spectroscopy will be required to resolve island contributions to impurity ion confinement.

Additionally, in a simple impurity force balance, helium is treated as a trace impurity in terms of the friction and thermal force effects. This results in inward and outward transport coefficients which are coupled to different leading order transport mechanisms for the trace impurity as compared to the main species. The magnetic island in the plasma edge can alter parallel plasma profiles and hence change the ratio of the friction and thermal force, which might change the main ion and impurity ion transport. These profiles, however, were experimentally not accessible and modeling with EMC3-EIRENE for these helium cases is left for future work.

These helium confinement measurements can be compared to recent measurements at both TEXTOR and $\mathrm{LHD}^{12,13}$. At these experiments, it was found that by using RMP fields, an island inserted into the edge region decreased the helium decay time (i.e. shorter values of $\left.\tau_{p, H e}^{*}\right)$. It was shown that at both of these experiments, these edge manipulations enhanced the outward transport of helium particles. The driving mechanism was found to be due to an increase of the outward directed friction force relative to the inward directed thermal force due to collisionality gradients. However, it has to be noted that for these experiments the islands did not influence the plasma wall interaction; rather, the islands were located inside of the confinement region in the helium ionized particle domain. In contrast, at W7$\mathrm{X}$ and HSX in the configurations with larger $\tau_{p, H e}^{*}$ (the Increased Iota and Edge-Islands configurations), the edge islands heavily influenced the plasma wall interaction and were present in the ionization source region (at HSX).

\section{Final considerations and conclusions}

As a final note, it is important to consider if the changes to the plasma core (i.e. through neoclassical transport) and that those changes, rather than edge structure induced changes, contributed to these measured differences $\tau_{p}^{*}$ and $\tau_{p}$ at both HSX and W7-X. In particular a possible increase of the effective ripple to adjust the edge structure $\left(\epsilon_{\text {eff }}\right.$ on both W7-X and HSX was increased by a factor of 2) may have had deleterious effects on the core transport in the Increased Iota configuration and the No-Edge-Islands configuration. However, the HSX ions are in the Plateau regime ${ }^{30}$ and the W7-X ions are in the Plateau/Pfirsch-Schületer regimes. In these regimes, there is no explicit transport dependence on the effective ripple. This means that the $\tau_{p}^{*}$ helium decay measurements (produced from ion emission) do not reflect any differences in core transport. With regard to the electrons, it was found that there were no major differences in core electron transport between the Standard configuration and Increased Iota configurations at W7-X due to the mitigating presence of a positive radial electric field ${ }^{55,56}$. At HSX the role of the radial 
electric field ${ }^{57,58}$ in mitigating transport is still an open question. Finally, EMC3-EIRENE simulations contain no information about potential core neoclassical transport changes that may have occurred as a result of the change in magnetic configuration (i.e. through the increased effective ripple). Despite this, EMC3-EIRENE results clearly show a difference in particle penetration as a result of changes in edge topology exclusively. These results demonstrate that the changes in our measured values of $\tau_{p}^{*}$ and $\tau_{p}$ stem primarily from changes in edge, rather than core, transport.

The edge magnetic structure in the HSX and W7-X stellarators was shown to have a significant impact on the particle fueling and exhaust of the plasma main species (hydrogen) as well as impurity helium. In HSX, the 8/7 island chain located just outside the LCFS was shown to reduce particle penetration and therefore increase global effective and true particle confinement times for both hydrogen and helium. These changes were possible because the magnetic perturbations took place within the plasma ionization domain. In contrast at W7-X, the movement of the $5 / 6$ island inwards and the introduction of the $5 / 6$ into the edge does not result in any significant changes in hydrogen particle confinement times, likely because neither island chain was located in the ionization source region. However, an increase in the effective helium particle confinement time was observed when the $5 / 5$ island was present in the edge, suggesting that the change in edge topology increased helium confinement to some degree. These findings suggest that for a helically optimized system like HSX, the plasma fueling from the recycling source as well as from active gas injection can be controlled by the magnetic island chain in the plasma edge.

\section{ACKNOWLEDGMENTS}

This work was supported in part by the U.S. Department of Energy (DOE) under grants DE-SC0014210, DEFG02-93ER54222, DE-AC05-00OR22725, DOE LANS Contract DE-AC52-06NA25396. The publisher, by accepting the article for publication acknowledges, that the United States Government retains a non-exclusive, paidup, irrevocable, world-wide license to publish or reproduce the published form of this manuscript, or allow others to do so, for United States Government purposes. This work has been carried out within the framework of the EUROfusion Consortium and has received funding from the Euratom research and training programme 2014-2018 under grant agreement No 633053. The views and opinions expressed herein do not necessarily reflect those of the European Commission.

\footnotetext{
${ }^{1}$ Y. Feng, F. Sardei, P. Grigull, K. McCormick, J. Kisslinger, and D. Reiter, Nuclear Fusion 46, 807 (2006).

${ }^{2}$ F. Sardei, Y. Feng, P. Grigull, G. Herre, D. Hildebrandt, J. Hofmann, J. Kisslinger, R. Brakel, J. Das, and J. Geiger, Journal of nuclear materials 241, 135 (1997).

${ }^{3}$ J. Wesson and D. J. Campbell, Tokamaks, Vol. 149 (Oxford University Press, 2011).
}

${ }^{4}$ S. Hudson, D. Monticello, and A. Reiman, Physics of Plasmas 8, 3377 (2001).

${ }^{5}$ P. C. Stangeby, The plasma boundary of magnetic fusion devices, Plasma physics series (Institute of Physics Pub, Bristol ; Philadelphia, 2000).

${ }^{6}$ R. König, P. Grigull, K. McCormick, Y. Feng, J. Kisslinger, A. Komori, S. Masuzaki, K. Matsuoka, T. Obiki, and N. Ohyabu, Plasma physics and controlled fusion 44, 2365 (2002).

${ }^{7}$ Y. Feng, M. Kobayashi, T. Lunt, and D. Reiter, Plasma physics and controlled fusion 53, 024009 (2011).

${ }^{8}$ K. Ida, N. Ohyabu, T. Morisaki, Y. Nagayama, S. Inagaki, K. Itoh, Y. Liang, K. Narihara, A. Y. Kostrioukov, and B. Peterson, Physical review letters 88, 015002 (2001).

${ }^{9}$ K. Ida, S. Inagaki, N. Tamura, T. Morisaki, N. Ohyabu, K. Khlopenkov, S. Sudo, K. Watanabe, M. Yokoyama, and T. Shimozuma, Nuclear fusion 44, 290 (2004).

${ }^{10}$ K. Ida, S. Inagaki, M. Yoshinuma, N. Tamura, T. Morisaki, and LHD Experiment Group, Fusion Science and Technology 58, 113 (2010).

${ }^{11}$ A. R. Akerson, A. Bader, C. C. Hegna, O. Schmitz, L. A. Stephey, D. T. Anderson, F. S. B. Anderson, and K. M. Likin, Plasma Physics and Controlled Fusion 58, 084002 (2016).

${ }^{12}$ O. Schmitz, K. Ida, M. Kobayashi, A. Bader, S. Brezinsek, T. Evans, H. Funaba, M. Goto, O. Mitarai, and T. Morisaki, Nuclear Fusion 56, 106011 (2016).

${ }^{13}$ A. Bader, M. Kobayashi, O. Schmitz, A. Akerson, F. Effenberg, H. Frerichs, Y. Feng, C. Hegna, K. Ida, and LHD Experimental Group, Plasma Physics and Controlled Fusion 58, 124006 (2016).

${ }^{14}$ K. Ida, M. Yoshinuma, M. Goto, O. Schmitz, S. Dai, A. Bader, M. Kobayashi, G. Kawamura, C. Moon, and Y. Nakamura, Plasma Physics and Controlled Fusion 58, 74010 (2016).

${ }^{15}$ D. Anderson, A. Almagri, F. Anderson, P. Matthews, J. Shohet, and J. Talmadge, Bull. Am. Phys. Soc 39, 1601 (1994).

${ }^{16}$ G. Grieger and I. Milch, Physikalische Blätter 49, 1001 (1993).

${ }^{17}$ A. Galeev and R. Sagdeev (1979) p. 257.

${ }^{18}$ A. F. Almagri, D. T. Anderson, F. S. B. Anderson, P. H. Probert, J. L. Shohet, and J. N. Talmadge, IEEE Transactions on Plasma Science 27, 114 (1999).

${ }^{19}$ H. Hölbe, T. Sunn Pedersen, J. Geiger, S. Bozhenkov, R. König, Y. Feng, J. Lore, A. Lumsdaine, and the Wendelstein 7-X Team, Nuclear Fusion 56, 026015 (2016).

${ }^{20}$ H. F. Dylla, P. H. LaMarche, M. Ulrickson, R. J. Goldston, D. B. Heifetz, K. W. Hill, and A. T. Ramsey, Nuclear fusion 27, 1221 (1987).

${ }^{21}$ D. Hillis, K. Finken, J. Hogan, K. Dippel, R. Moyer, A. Pospieszczyk, D. Rusbüldt, K. Akaishi, R. Conn, and H. Euringer, Physical review letters 65, 2382 (1990).

${ }^{22}$ O. Schmitz, J. Coenen, H. Frerichs, M. Kantor, M. Lehnen, B. Unterberg, S. Brezinsek, M. Clever, T. Evans, K. Finken, M. Jakubowski, A. Kraemer-Flecken, V. Phillips, D. Reiter, U. Samm, G. Spakman, and G. Telesca, Journal of Nuclear Materials 390-391, 330 (2009).

${ }^{23}$ V. Soukhanovskii, R. Maingi, R. Raman, H. Kugel, B. LeBlanc, A. Roquemore, C. Skinner, and NSTX Research Team, Journal of nuclear materials 313, 573 (2003).

${ }^{24}$ R. Maingi, G. L. Jackson, M. R. Wade, M. A. Mahdavi, P. K. Mioduszewski, G. Haas, M. J. Schaffer, J. T. Hogan, and C. C. Klepper, Nuclear fusion 36, 245 (1996).

${ }^{25}$ D. L. Brower, C. Deng, W. X. Ding, D. T. Anderson, and W. Mason, Review of Scientific Instruments 72, 1081 (2001).

${ }^{26}$ K. Zhai, F. S. B. Anderson, K. Willis, K. Likin, and D. T. Anderson, Review of Scientific Instruments 75, 3900 (2004).

${ }^{27}$ S. Gerhardt, J. Canik, D. Anderson, and L. Owen, Review of scientific instruments 75, 2981 (2004).

${ }^{28}$ C. Deng, D. L. Brower, W. Ding, A. F. Almagri, D. T. Anderson, F. S. B. Anderson, S. P. Gerhardt, P. Probert, and J. N. Talmadge, Review of scientific instruments 74, 1625 (2003).

${ }^{29} \mathrm{~J}$. Winter, Plasma Physics and Controlled Fusion 38, 1503 (1996).

${ }^{30} \mathrm{~S} . \mathrm{P}$. Gerhardt, Measurements and modeling of the plasma re- 
sponse to electrode biasing in the hsx stellarator, phdthesis, Univeristy of Wisconsin - Madison (2004).

${ }^{31}$ D. Heifetz, D. Post, M. Petravic, J. Weisheit, and G. Bateman, Journal of Computational Physics 46, 309 (1982).

${ }^{32}$ H. Frerichs, D. Reiter, O. Schmitz, T. Evans, and Y. Feng, Nuclear Fusion 50, 034004 (2010).

${ }^{33}$ J. M. Canik, D. T. Anderson, F. S. B. Anderson, C. Clark, K. M. Likin, J. N. Talmadge, and K. Zhai, Physics of Plasmas 14, 056107 (2007).

${ }^{34}$ S. A. Bozhenkov, F. Effenberg, Y. Feng, J. Geiger, D. A. Hartmann, H. Hölbe, T. S. Pederson, and R. Wolf, in EPS Conference on Plasma Physics (2014).

${ }^{35}$ T. Sunn Pedersen, T. Andreeva, H.-S. Bosch, S. Bozhenkov, F. Effenberg, M. Endler, Y. Feng, D. Gates, J. Geiger, D. Hartmann, H. Hölbe, M. Jakubowski, R. König, H. Laqua, S. Lazerson, M. Otte, M. Preynas, O. Schmitz, T. Stange, Y. Turkin, and the W7-X Team, Nuclear Fusion 55, 126001 (2015).

${ }^{36}$ M. Krychowiak, A. Adnan, A. Alonso, T. Andreeva, J. Baldzuhn, T. Barbui, M. Beurskens, W. Biel, C. Biedermann, B. D. Blackwell, H. S. Bosch, S. Bozhenkov, R. Brakel, T. Bräuer, B. B. d. Carvalho, R. Burhenn, B. Buttenschön, A. Cappa, G. Cseh, A. Czarnecka, A. Dinklage, P. Drews, A. Dzikowicka, F. Effenberg, M. Endler, V. Erckmann, T. Estrada, O. Ford, T. Fornal, H. Frerichs, G. Fuchert, J. Geiger, O. Grulke, J. H. Harris, H. J. Hartfuß, D. Hartmann, D. Hathiramani, M. Hirsch, U. Höfel, S. Jabłoński, M. W. Jakubowski, J. Kaczmarczyk, T. Klinger, S. Klose, J. Knauer, G. Kocsis, R. König, P. Kornejew, A. Krämer-Flecken, N. Krawczyk, T. Kremeyer, I. Ksiażek, M. Kubkowska, A. Langenberg, H. P. Laqua, M. Laux, S. Lazerson, Y. Liang, S. C. Liu, A. Lorenz, A. O. Marchuk, S. Marsen, V. Moncada, D. Naujoks, H. Neilson, O. Neubauer, U. Neuner, H. Niemann, J. W. Oosterbeek, M. Otte, N. Pablant, E. Pasch, T. S. Pedersen, F. Pisano, K. Rahbarnia, L. Ryć, O. Schmitz, S. Schmuck, W. Schneider, T. Schröder, H. Schuhmacher, B. Schweer, B. Standley, T. Stange, L. Stephey, J. Svensson, T. Szabolics, T. Szepesi, H. Thomsen, J.-M. Travere, H. T. Mora, H. Tsuchiya, G. M. Weir, U. Wenzel, A. Werner, B. Wiegel, T. Windisch, R. Wolf, G. A. Wurden, D. Zhang, A. Zimbal, and S. Zoletnik, Review of Scientific Instruments 87, 11D304 (2016).

${ }^{37}$ J. Knauer, P. Kornejew, H. T. Mora, M. Hirsch, A. Werner, and R. Wolf, in EPS Conference on Plasma Physics (European Physical Society, 2016)

${ }^{38}$ L. Stephey, G. A. Wurden, O. Schmitz, H. Frerichs, F. Effenberg, C. Biedermann, J. Harris, R. König, P. Kornejew, M. Krychowiak, E. A. Unterberg, and the W7-X Team, Review of Scientific Instruments 87, 11D606 (2016).

${ }^{39}$ R. Colchin, D. Hillis, R. Maingi, C. Klepper, and N. Brooks, Review of scientific instruments 74, 2068 (2003).

${ }^{40}$ N. H. Brooks, R. J. Colchin, D. T. Fehling, D. L. Hillis, Y. Mu, and E. Unterberg, Review of Scientific Instruments 79, 10F330 (2008).

${ }^{41}$ E. A. Unterberg, O. Schmitz, D. H. Fehling, H. Stoschus, C. C. Klepper, J. M. Muñoz-Burgos, G. Van Wassenhove, and D. L. Hillis, Review of Scientific Instruments 83, 10D722 (2012).

${ }^{42}$ F. Effenberg, Y. Feng, O. Schmitz, H. Frerichs, S. Bozhenkov, H. Hölbe, R. König, M. Krychowiak, T. Sunn Pedersen, D. Reiter, L. Stephey, and W.-X. Team, Nuclear Fusion 57, 036021 (2017).

${ }^{43}$ S. Brezinsek, P. T. Greenland, P. Mertens, A. Pospieszczyk, D. Reiter, U. Samm, B. Schweer, and G. Sergienko, Journal of nuclear materials 313, 967 (2003).
${ }^{44}$ T. Szepesi, A. Alonso, C. Biedermann, G. Cseh, A. Dinklage, M. Jakubowski, G. Kocsis, R. König, M. Otte, and T. Sunn Pedersen, in EPS Conference on Plasma Physics (European Physical Society, 2016).

${ }^{45}$ T. Barbui, M. Krychowiak, R. König, O. Schmitz, J. M. Burgos, B. Schweer, and A. Terra, Review of Scientific Instruments 87, 11E554 (2016)

${ }^{46}$ J. Ehrenberg, P. Coad, L. De Kock, S. Erents, A. Gondhalekar, D. Goodall, J. Hancock, P. Harbour, T. Jones, and G. McCracken, Journal of Nuclear Materials 162, 63 (1989).

${ }^{47}$ A. Bader, D. Anderson, C. Hegna, Y. Feng, J. Lore, and J. Talmadge, Nuclear Fusion 53, 113036 (2013).

${ }^{48}$ I. Waters, H. Frerichs, R. Akers, Y. Feng, K. Flesch, A. Kirk, D. Ryan, O. Schmitz, and A. Thornton (2017).

${ }^{49}$ L. Stephey, A. Bader, D. Anderson, J. Talmadge, C. Hegna, and F. Anderson, in APS Division of Plasma Physics Meeting (2012).

${ }^{50}$ R. Majeski, R. Doerner, T. Gray, R. Kaita, R. Maingi, D. Mansfield, J. Spaleta, V. Soukhanovskii, J. Timberlake, and L. Zakharov, Physical review letters 97, 075002 (2006).

${ }^{51} \mathrm{~J}$. Cordey, D. Bartlett, and R. Bickerton, Particle and energy confinement in Ohmically heated JET plasmas (IAEA, International Atomic Energy Agency (IAEA), 1985).

${ }^{52}$ E. S. Marmar, Journal of Nuclear Materials 76, 59 (1978).

${ }^{53}$ W. L. Rowan, C. C. Klepper, C. P. Ritz, R. D. Bengtson, K. W. Gentle, P. E. Phillips, T. L. Rhodes, B. Richards, and A. J. Wootton, Nuclear fusion 27, 1105 (1987).

${ }^{54}$ R. Freeman and E. M. Jones, "Atomic collision processes in plasma physics experiments," Tech. Rep. (UKAEA Research Group, 1974).

${ }^{55}$ A. Dinklage, A. Alonso, J. Baldzuhn, C. D. Beidler, C. Biedermann, B. Blackwell, S. Bozhenkov, R. Brakel, B. Buttenschön, Y. Feng, G. Fuchert, J. Geiger, P. Helander, M. Hirsch, U. Hoefel, J. Knauer, A. Kramer-Flecken, M. Landreman, A. Langenberg, H. P. Laqua, H. Maassberg, N. Pablant, E. Pasch, K. Rahbarnia, L. Rudischhauser, H. Smith, T. Stange, L. Stephey, H. Trimino-Mora, Y. Turkin, J.-L. Velasco, G. Wurden, D. Zhang, T. Andreeva, M. Beurskens, E. Blanco, H.S. Bosch, T. Fornal, O. Grulke, D. Hartmann, J. Harris, M. Jakubowski, T. Klinger, S. Klose, G. Kocsis, R. Koenig, P. Kornejew, N. Krawczyk, M. Krychowiak, M. Kubkowska, I. Ksiazek, S. Lazerson, Y. Liang, S. Liu, O. Marchuk, S. Marsen, N. Marushchenko, V. Moncada, D. Moseev, D. Naujoks, H. Niemann, M. Otte, T. Pedersen, F. Pisano, K. Risse, T. Rummel, O. Schmitz, S. Satake, T. Schroeder, T. Szepesi, H. Thomsen, P. Traverso, H. Tsuchiya, P. Valson, N. Wang, T. Wauters, G. Weir, R. Wolf, M. Yokoyama, and the W7-X Team, in EPS Conference on Plasma Physics (2016).

${ }^{56}$ N. Pablant, S. Bozhenkov, A. Dinklage, G. Fuchert, M. Landreman, A. Langenberg, A. Alonso, C. D. Beidler, M. Beurskens, M. Bitter, R. Burhenn, L. Delgado-Aparicio, D. Gates, J. Geiger, K. W. Hill, M. Hirsch, U. Hoefel, J. Knauer, A. Kramer-Flecken, S. Lazerson, H. Maassberg, O. Marchuk, N. Marushchenko, J. Mittelstaedt, D. R. Mikkelsen, E. Pasch, S. Satake, H. Smith, J. Svensson, P. Traverso, Y. Turkin, P. Valson, J. Velasco, G. M. Weir, T. Windisch, R. Wolf, M. Yokoyama, and D. Zhang, in APS Division of Plasma Physics Meeting (2016).

${ }^{57}$ S. Kumar, J. Talmadge, T. Dobbins, F. Anderson, K. Likin, and D. Anderson, submitted to Nuclear Fusion, NF-101261.R1 (2016).

58 A. Briesemeister, K. Zhai, D. Anderson, F. Anderson, and J. Talmadge, Plasma Physics and Controlled Fusion 55, 014002 (2012). 